TRANSACTIONS OF THE

AMERICAN MATHEMATICAL SOCIETY

Volume 355, Number 8, Pages 3117-3141

S 0002-9947(03)03295-1

Article electronically published on April 14, 2003

\title{
D-LOG AND FORMAL FLOW FOR ANALYTIC ISOMORPHISMS OF N-SPACE
}

\author{
DAVID WRIGHT AND WENHUA ZHAO
}

\begin{abstract}
Given a formal map $F=\left(F_{1}, \ldots, F_{n}\right)$ of the form $z+$ higher-order terms, we give tree expansion formulas and associated algorithms for the D-Log of $F$ and the formal flow $F_{t}$. The coefficients that appear in these formulas can be viewed as certain generalizations of the Bernoulli numbers and the Bernoulli polynomials. Moreover, the coefficient polynomials in the formal flow formula coincide with the strict order polynomials in combinatorics for the partially ordered sets induced by trees. Applications of these formulas to the Jacobian Conjecture are discussed.
\end{abstract}

\section{INTRODUCTION}

This work began as an effort to link and extend the results of [W2] and [Z], placing them in a common framework. Both of these papers deal with the formal inverse $F^{-1}$ of a system of power series $F=\left(F_{1}, \ldots, F_{n}\right)$; both give formulas for $F^{-1}$ in terms of $F$, the former being a tree formula, the latter an exponential formula. This quest has led to a host of interesting connections, algorithms, formulas, and relationships with combinatorics, Bernoulli numbers, and Bernoulli polynomials.

The former paper deals with tree formulas as they apply to formal inverse, a thread which is also the main thrust of [BCW], [W1, [W2, and [CMTWW]. It has combinatoric connections with generating functions and enumeration techniques for trees. The general goal of power series inversion (sometimes called "reversion", perhaps to distinguish functional inverse from multiplicative inverse) is as follows. Let $F=\left(F_{1}, \ldots, F_{n}\right)$ with $F_{i} \in \mathbb{C}\left[\left[z_{1}, \ldots, z_{n}\right]\right]$ for each $i$ and $F_{i}=z_{1}+$ terms of degree $\geq 2$. One seeks formulas for the unique $G_{1}, \ldots, G_{n} \in$ $\mathbb{C}\left[\left[z_{1}, \ldots, z_{n}\right]\right]$ for which $G_{i}(F)=z_{i}$, for $i=1, \ldots, n$. Perhaps the first of these was the Lagrange Inversion Formula (see [St2], Chapter 5), which dealt with the case $n=1$, and which was generalized (under a certain restrictive hypothesis) to all $n$ by I. J. Good [Go in 1960. Good then uses his formula for problems of enumerating certain trees. In fact, Good's formula had been discovered and published by Jacobi in 1830 [Ja]. Another paper which appeared in 1960 was that of G. N. Raney $[\mathrm{R}$, who also related formal inverse to trees. Raney's work was generalized in CMTWW, which also utilized the work of Jacobi. A general inversion formula was given by Abhyankar and Gurjar in 1974 [A], and this is the source from which

Received by the editors June 5, 2002 and, in revised form, January 3, 2003.

2000 Mathematics Subject Classification. Primary 14R10, 11B68; Secondary 14R15, 05C05.

Key words and phrases. D-log, formal flow of automorphisms, rooted trees, order polynomials, Bernoulli numbers and polynomials. 
the tree formula of [BCW] was derived, with the hope of applying it to the Jacobian Conjecture. Other treatments of the subject of inversion are [HS], [Ge], and [Jo].

The tree formula of $[\mathrm{BCW}]$ expresses the formal inverse $F^{-1}$ as an infinite $\mathbb{Q}$ linear combination of certain power series $\mathcal{P}_{T} \in \mathbb{C}[[z]]$, which are constructed using finite rooted trees $T$. This construction will be reviewed in $\S 2$ and a new (and quick) proof of the inversion formula, using the tools developed in this paper, will be presented in $\S 5$ (Theorem 5.1).

Amongst the results of the latter paper is the realization of $F$ by an expression $F=\exp (A) \cdot z$, where $A=A(z)$, called the $D$ - $\log$ of $F$, is a differential operator uniquely determined by $F$ and yielding the formal inverse as $F^{-1}=\exp (-A) \cdot z$. Furthermore, the formal flow $F_{t}=\exp (t A) \cdot z$ encodes all powers $F^{[n]}$ with $n \in \mathbb{Z}$ of the formal map $F$. The D-Log and the formal flow will be reviewed in $\S 3$.

A primary goal was to show that the D-Log $A$ can also be expressed as a $\mathbb{Q}$-linear combination of the power series $\mathcal{P}_{T}$. This goal was attained, yielding a tree formula for the D-Log. Moreover, we discovered that the rational coefficients $\phi_{T}$ of this expression can be generated by an elegant recurrence relationship and possess some intriguing combinatorial properties. For example, the Bernoulli numbers appear amongst these coefficients.

This situation is placed in a larger context which incorporates formal inverse by considering the formal flow $F_{t}=\exp (t A) \cdot z$, where $t$ is an indeterminate. For $n \in \mathbb{N}$, setting $t=n$ gives the $n$-fold composition $F \circ \cdots \circ F$, and setting $t=-n$ gives the $n$-fold composition $F^{-1} \circ \cdots \circ F^{-1}$. The system $F_{t}$ can be written as a $\mathbb{Q}[t]$-linear combination of the power series $\mathcal{P}_{T}$, producing for each rooted tree $T$ a polynomial $\psi_{T}(t)$ having $\phi_{T}$ as the coefficient of $t$. Among these polynomials are the binomial polynomials $\left(\begin{array}{c}t \\ m\end{array}\right)$, for all positive integers $m$. We give an algorithm for calculating $\psi_{T}(t)$ using the difference operator $\Delta$. This formula is used to establish the relationship of certain $\psi_{T}(t)$ 's with the Bernoulli polynomials $B_{m}(t)$ via an integration formula. It is shown that $\psi_{T}(t)$ also provides an interesting combinatorial connection: It coincides with the strict order polynomial $\bar{\Omega}(P, t)$ (see St1, Chapter 4) for $P=T$, which, for $t=m \in \mathbb{Z}^{+}$, counts the number of strict order-preserving maps from any partially ordered set $P$ to the totally ordered set with $m$ elements.

Acknowledgments. We would like to thank Professor John Shareshian for informing us of Theorem 4.5, and also Professor Steve Krantz for a helpful conversation on flow of analytic maps.

\section{Tree Operations}

2.1. Notation. By a rooted tree we mean a finite 1-connected graph with one vertex designated as its root. The 1-connectivity provides the notion of distance between two vertices, which is defined as the number of edges in the unique geodesic connecting the two. The height of a tree is defined to be the maximum distance of any vertex from the root. In a rooted tree there are natural ancestral relations between vertices. We say a vertex $w$ is a child of vertex $v$ if the two are connected by an edge and $w$ lies further from the root than $v$. In the same situation, we say $v$ is the parent of $w$. Note that a vertex may have several children, but only one parent. The root is the only vertex with no parent. A vertex is called a leaf if it has no children. When we speak of isomorphisms between rooted trees, we will always mean root-preserving isomorphisms. 
With these notions in mind, we establish the following notation.

(1) We let $\mathbb{T}$ be the set of isomorphism classes of all rooted trees and, for $m \geq 1$ an integer, we let $\mathbb{T}_{m}$ be the set of isomorphism classes of all rooted trees with $m$ vertices. The latter is a finite set.

(2) For any rooted tree $T$, we set the following notation:

- $\mathrm{rt}_{T}$ denotes the root vertex of $T$.

- $E(T)$ denotes the set of edges of $T$.

- $V(T)$ denotes the set of vertices of $T$.

- $L(T)$ denotes the set of leaves of $T$.

- $v(T)$ (resp. $l(T))$ denotes the number of the elements of $V(T)$ (resp. $L(T))$.

- $h(T)$ denotes the height of $T$.

- $\alpha_{T}$ denotes the number of the elements of the automorphism group $\operatorname{Aut}(T)$.

- For $v \in V(T)$ we denote by $\alpha_{T, v}$ the size of the stabilizer of $v$ in Aut $(T)$. Similarly, for $e \in E(T)$, we write $\alpha_{T, e}$ for the size of the stabilizer of $e$ in $\operatorname{Aut}(T)$.

- For $e \in E(T)$ we denote by $v_{e}$ and $v_{e}^{\prime}$ the two (distinct) vertices that are connected by $e$, with $v_{e}$ being the one closest to the root.

- For $v \in V(T)$ we denote by $v^{+}$the set of vertices that are children of $v$.

- For $v \in V(T)$ we define the height of $v$ to be the number of edges in the (unique) geodesic connecting $v$ to $\mathrm{rt}_{T}$. The height of $T$ is defined to be the maximum of the heights of its vertices.

- For $v_{1}, \ldots, v_{r} \in V(T)$, we write $T \backslash\left\{v_{1}, \ldots, v_{r}\right\}$ for the graph obtained by deleting each of these vertices and all edges adjacent to these vertices.

(3) A rooted subtree of a rooted tree $T$ is defined as a connected subgraph of $T$ containing $\mathrm{rt}_{T}$, with $\mathrm{rt}_{T^{\prime}}=\mathrm{rt}_{T}$. In this case we write $T^{\prime} \leq T$. If $T^{\prime} \neq T$, we write $T^{\prime}<T$. If $T^{\prime}<T$, we write $T \backslash T^{\prime}$ for the graph obtained by deleting all vertices of $T^{\prime}$ and all edges adjacent to its vertices.

(4) For any $k \geq 1$, we denote by $C_{k}$ the rooted tree of height $k-1$ having $k$ vertices, and by $S_{k}$ the rooted tree of height 1 having $k$ leaves. We also set $S_{0}=\circ$, the rooted tree with one vertex. We refer to the trees $C_{k}$ as chains and the $S_{k}$ as shrubs.

2.2. Power Series Given by a Rooted Tree. Let $\mathbb{C}\left[\left[z_{1}, \ldots, z_{n}\right]\right]=\mathbb{C}[[z]]$ denote the ring of formal power series in $n$ variables $z_{1}, \ldots, z_{n}$ over the complex number\$ $\mathbb{C}$. For $i=1, \ldots, n$ we will write $D_{i}$ for the differential operator $\frac{\partial}{\partial z_{i}}$. The operators $D_{1}, \ldots, D_{n}$ are commuting derivations acting on the ring $\mathbb{C}[[z]]$.

Given a vector of power series $F=\left(F_{1}, \ldots, F_{n}\right) \in \mathbb{C}[[z]]^{n}$, we write $F_{i}=z_{i}+H_{i}$ for $i=1, \ldots, n$, or just $F=z+H$ In most applications the power series $H=$ $\left(H_{1}, \ldots, H_{n}\right)$ will involve only monomials of total degree 2 and higher, and we will often take $H$ to be homogeneous of degree $d \geq 2$. However, these assumptions are

\footnotetext{
${ }^{1}$ In this paper $\mathbb{C}$ can always be replaced by any $\mathbb{Q}$-algebra.

${ }^{2}$ We should here acknowledge that in almost every other treatment of this subject the system $F$ is written as $z-H$, which yields nicer looking formulas for the formal inverse of $F$. The reason for our choice is that the formulas involving the D-Log and formal flow, which will be developed in $\S 3$, come out better when we write $F=z+H$.
} 
not necessary for what follows here. We will associate to each rooted tree a power series in $n$ variables based on $F$ (equivalently, on $H$ ).

For $T \in \mathbb{T}$, a labeling of $T$ in the set $\{1, \ldots, n\}$ is a function $f: V(T) \rightarrow$ $\{1, \ldots, n\}$. A rooted tree $T$ with a labeling $f$ is called a labeled rooted tree, denoted $(T, f)$. Given such, and given $F=z+H$ as above, we make the following definitions, for $v \in V(T)$ :

(1) $H_{v}=H_{f(v)}$;

(2) $D_{v}=D_{f(v)}$

(3) $D_{v^{+}}=\prod_{w \in v^{+}} D_{w}$;

(4) $P_{T, f}=\prod_{v \in V(T)} D_{v^{+}} H_{v}$.

Finally, we define systems of power series $P_{T}=\left(P_{T, 1}, \ldots, P_{T, n}\right)$ and $\mathcal{P}_{T}=\left(\mathcal{P}_{T, 1}, \ldots\right.$, $\mathcal{P}_{T, n}$ ) by summing over all labelings of $T$ having a fixed label for the root

$$
\begin{aligned}
P_{T, i} & =\sum_{\substack{f: V(T) \rightarrow\{1, \ldots, n\} \\
f\left(\mathrm{rt}_{T}\right)=i}} P_{T, f}, \\
\mathcal{P}_{T, i} & =\frac{1}{\alpha_{T}} P_{T, i}
\end{aligned}
$$

for $i=1, \ldots, n$.

One notes that the systems of power series $P_{T}$ and $\mathcal{P}_{T}$ are dependent on the integer $n$ and the system $H=\left(H_{1}, \ldots, H_{n}\right) \in \mathbb{C}[[z]]^{n}$. They can be viewed as objects which determine functions $\mathbb{C}\left[\left[z_{1}, \ldots, z_{n}\right]\right]^{n} \rightarrow \mathbb{C}\left[\left[z_{1}, \ldots, z_{n}\right]\right]^{n}$ for all $n \geq 1$. We will write $P_{T}(H)$ and $\mathcal{P}_{T}(H)$ when we need to emphasize this dependence, or when we are dealing with more than one system $H$.

2.3. Stable Linear Independence. We begin by establishing an important independence property of the objects $\left\{P_{T} \mid T \in \mathbb{T}\right\}$.

Definition 2.1. We say that rooted trees $T_{1}, \ldots, T_{k}$ are stably linearly dependent if there exist $c_{1}, \ldots, c_{k} \in \mathbb{C}$ such that $\sum_{i=1}^{k} c_{i} P_{T_{i}}=0$ for any integer $n \geq 1$ and any homogeneous polynomial system $H=\left(H_{1}, \ldots, H_{n}\right)$ in $n$ variables. Otherwise, we say that $T_{i}$ are stably linearly independent.

Remark 2.2. If $H$ is homogeneous of degree $d$ and if $T \in \mathbb{T}_{m}$, then $P_{T}(H)$ is homogeneous of degree $(d-1) m+1$. Thus if we partition $\left\{T_{1}, \ldots, T_{k}\right\}$ according to the number of vertices in a tree, then $T_{1}, \ldots, T_{k}$ are stably linearly independent if and only if each partition is a stably linearly independent set of trees.

Lemma 2.3. Suppose that $\sum_{i=1}^{k} c_{i} P_{T_{i}}(H)=0$ for any integer $n \geq 1$ and any homogeneous polynomial system $H$ in $n$ variables. Then $\sum_{i=1}^{k} c_{i} P_{T_{i}}(H)=0$ for any system of power series $H=\left(H_{1}, \ldots, H_{n}\right)$ in $n$ variables.

Proof. We first prove it for any polynomial $H$ (not necessarily homogeneous) in $n$ variables by introducing a new variable $z_{n+1}$ and homogenizing $H$ using $z_{n+1}$. Call the resulting homogeneous system $\bar{H}$. Setting $\widetilde{H}=\left(\bar{H}, H_{n+1}=0\right)$, we have

$$
\sum_{i=1}^{k} c_{i} P_{T_{i}}(H, z)=\left.\sum_{i=1}^{k} c_{i} P_{T_{i}}(\widetilde{H}, z)\right|_{z_{n+1}=1}=0,
$$

which proves the lemma for $H$ a polynomial system. For an arbitrary system of power series $H$ we note that if $T$ is a tree with $r$ edges, the homogeneous summands 
of degree $\leq d$ in $P_{T}(H)$ depend only on the homogeneous summands of $H$ having degree $\leq d+r$. Taking $r$ to be the maximum of the numbers of edges in $T_{1}, \ldots, T_{k}$, then all terms of degree $\leq d$ in $\sum_{i=1}^{k} c_{i} P_{T_{i}}(H)$ depend only the homogeneous summands of $H$ having degree $\leq d+r$. Taking $\widehat{H}$ to be the polynomial truncation of $H$ of degree $d+r$, we see that $\sum_{i=1}^{k} c_{i} P_{T_{i}}(H)$ and $\sum_{i=1}^{k} c_{i} P_{T_{i}}(\widehat{H})$ coincide up through degree $d$. Since the latter is zero ( $\widehat{H}$ being a polynomial system) and $d$ is arbitrary, we must have $\sum_{i=1}^{k} c_{i} P_{T_{i}}(H)=0$.

Theorem 2.4. Any rooted trees $T_{i}(i=1,2, \ldots, k)$ with $T_{i} ¥ T_{j}$ for any $i \neq j$ are stably linearly independent.

Before giving the proof we will define a polynomial system depending on a rooted tree. Given a rooted tree $T$ with $m$ vertices, we create variables $z_{1}, \ldots, z_{m}$. Label the edges $e_{2}, \ldots, e_{m}$ and assign each variable $z_{i}$ with $2 \leq i \leq m$ to the edge $e_{i}$. Label the vertices as follows: $v_{1}=\mathrm{rt}_{T}$, and for $i=2, \ldots, m$ let $v_{i}$ be the vertex of $e_{i}$ that is farthest from the root. For each vertex $v_{i} \in V(T)$, we define $H_{i}$ to be the product of all the variables assigned to the edges connecting $v_{i}$ with its children. (Thus if $v_{i}$ is a leaf, we have $H_{i}=1$.) Set $H_{T}=\left(H_{1}, \ldots, H_{m}\right)$. We have

Lemma 2.5. Let $T$ and $T^{\prime}$ be two rooted trees with the same number of vertices. Then

$$
P_{T^{\prime}}\left(H_{T}\right)=\left\{\begin{array}{lr}
(0, \ldots, 0) & \text { if } T \supsetneqq T^{\prime}, \\
\left(\alpha_{T}, 0, \ldots, 0\right) & \text { if } T \cong T^{\prime} .
\end{array}\right.
$$

Proof. The following facts are not difficult to verify and provide a sketch of the proof: Each coordinate $H_{T, i}$ of $H_{T}$ is a monomial that is linear or constant with respect to each variable $z_{i}$. Each coordinate is constant with respect to $z_{1}$. Each variable $z_{i}$ with $i \geq 2$ appears in precisely one coordinate $H_{T, j}$, and $i \neq j . P_{T^{\prime}}\left(H_{T}\right)$ is a homogeneous system of degree zero, and must be equal to either 0 or 1 . If a labeling $f: V\left(T^{\prime}\right) \rightarrow\{1, \ldots, m\}$ is not bijective, then $P_{T^{\prime}, f}=0$ since it would entail differentiating two different coordinates $H_{T, i}$ and $H_{T, j}$ with respect to the same variable, or differentiating some $H_{T, i}$ twice by the same variable, or differentiating some $H_{T, i}$ by $z_{i}$, all of which give zero. Moreover, if $f\left(\mathrm{rt}_{T^{\prime}}\right) \neq 1$, then $P_{T^{\prime}, f}=0$, since it would entail differentiation by $z_{1}$, and therefore $P_{T^{\prime}}\left(H_{T}\right)$ is zero except possibly in the first coordinate.

With this it is not hard to show that, if $f: V\left(T^{\prime}\right) \rightarrow\{1, \ldots, m\}$ is a labeling for which $P_{T^{\prime}, f} \neq 0$, then the function $V\left(T^{\prime}\right) \rightarrow V(T)$ defined by $w \mapsto v_{f(w)}$ gives an isomorphism of $\varphi: T^{\prime} \rightarrow T$. Finally, the group Aut $T$ acts freely and transitively on the set of labelings $f: V(T) \rightarrow\{1, \ldots, m\}$ for which $P_{T, f} \neq 0$. The lemma follows easily from these statements.

Proof of Theorem 2.4. Suppose that $\sum_{i=1}^{k} c_{i} P_{T_{i}}(z)=0$ with $c_{1} \neq 0$. Choose $H=$ $H_{T_{1}}$. Then there must exist $j \neq 1$ such that $P_{T_{j}}\left(H_{T_{1}}\right) \neq 0$. By the lemma above, we have $T_{1} \cong T_{j}$.

If $H=\left(H_{1}, \ldots, H_{n}\right)$ is a system of power series such that each $H_{i}$ has only terms of degree $d$ and higher, the power series $\mathcal{P}_{T}$ has only terms of degree $(d-1) v(T)+1$ and higher. Hence if $d \geq 2$, a sum of the form $\sum_{T \in \mathbb{T}} c_{T} \mathcal{P}_{T}$ makes sense, since only finitely many terms contribute to any specified homogeneous summand. With this observation, we state the following consequence of stable linear independence. 
Corollary 2.6. Suppose we have a collection $\left\{c_{T}\right\} \subset \mathbb{C}$ indexed by the rooted trees $T \in \mathbb{T}$ such that $\sum_{T \in \mathbb{T}} c_{T} \mathcal{P}_{T}=0$ for any integer $n \geq 1$ and any system of power series $H=\left(H_{1}, \ldots, H_{n}\right)$ with $H$ having only terms of degree $\geq 2$. Then $c_{T}=0$ for all $T \in \mathbb{T}$.

Proof. We consider systems $H$ that are homogeneous polynomial systems of degree $d \geq 2$. In this case $\mathcal{P}_{T}$ is homogeneous of degree $(d-1) v(T)+1$. So the homogeneous summands of $\sum_{T \in \mathbb{T}} c_{T} \mathcal{P}_{T}$ are the finite sums $\sum_{T \in \mathbb{T}_{N}} c_{T} \mathcal{P}_{T}$ for $N \in \mathbb{N}$; so these must be zero. By Theorem 2.4 applied to the finite set of trees $\mathbb{T}_{N}$, we must have $c_{T}=0$ for all $T \in \mathbb{T}_{N}$.

Recall that we are writing $D_{i}$ for the operator $\frac{\partial}{\partial z_{i}}$. We will denote by $D$ the column vector $\left(D_{1}, \ldots, D_{n}\right)^{\mathrm{t}}$. We now define a differential operator on $\mathbb{C}[[z]]$ for each $T \in \mathbb{T}$.

Definition 2.7. For $T \in \mathbb{T}$, we denote by $D_{T}$ the differential operator $P_{T} D=$ $\sum_{i=1}^{n} P_{T, i} D_{i}$. We will write $\mathcal{D}_{T}$ for the operator $\mathcal{P}_{T} D=\frac{1}{\alpha_{T}} D_{T}$.

2.4. Tree Surgery. We will now discuss some "surgical" procedures on trees. Given $T \in \mathbb{T}$ and $e \in E(T)$, the removal of the edge $e$ from $T$ gives a disconnected graph with two connected components which are trees. We denote by $T_{e}$ the component containing $\mathrm{rt}_{T}$, and by $T_{e}^{\prime}$ the other component. We give $T_{e}$ and $T_{e}^{\prime}$ the structure of rooted trees by setting $\mathrm{rt}_{T_{e}}=\mathrm{rt}_{T}$ and $\mathrm{rt}_{T_{e}^{\prime}}=v_{e}^{\prime}$.

Given rooted trees $T$ and $T^{\prime}$ and $v \in V(T)$, we denote by

$$
T^{\prime} \multimap_{v} T
$$

the tree obtained by connecting $\mathrm{rt}_{T^{\prime}}$ and $v$ by a newly created edge, and setting $\mathrm{rt}_{\left(T^{\prime}-_{v} T\right)}=\mathrm{rt}_{T}$. We will refer to the newly created edge as the connection edge of $T^{\prime} \multimap_{v} T$. Note that for any tree $T$ and edge $e \in E(T)$ we have an obvious isomorphism $T \cong\left(T_{e}^{\prime} \multimap v_{e} T_{e}\right)$ which is the identity on $T_{e}$ and $T_{e}^{\prime}$.

Given $e, f \in E(T)$, we say " $f$ lies below $e$ ", and write $e \succ f$, if $f \in E\left(T_{e}\right)$. This merely says that $f$ remains when we "strip away" $e$ and $T_{e}^{\prime}$. One can easily see that this relation is not transitive. However, if we write

$$
e_{1} \succ \cdots \succ e_{r},
$$

for $e_{1}, \ldots, e_{r} \in E(T)$, we will mean by this that $e_{i} \succ e_{j}$ if $i<j$.

A sequence $\vec{e}=\left(e_{1}, \ldots, e_{r}\right) \in E(T)^{r}$ with $e_{1} \succ \cdots \succ e_{r}$ determines a sequence of subtrees $T_{\vec{e}, 1}, \ldots T_{\vec{e}, r+1}$ as follows: Set $T_{\vec{e}, 1}=T_{e_{1}}^{\prime}$ and let $S_{2}=T_{e_{1}}$, noting that $e_{2}, \ldots, e_{r} \in E\left(S_{2}\right)$. For $i=1, \ldots, r$, assume that $T_{\vec{e}, 1}, \ldots, T_{\vec{e}, i-1}, S_{i}$ are defined with $e_{i}, \ldots, e_{r} \in E\left(S_{i}\right)$. Set $T_{\vec{e}, i}=\left(S_{i}\right)_{e_{i}}^{\prime}$ and $S_{i+1}=\left(S_{i}\right)_{e_{i}}$. Finally, set $T_{\vec{e}, r+1}=S_{r+1}$.

For any integer $r \geq 1$ and $T \in \mathbb{T}$, create an indeterminate $Y_{T}^{(r)}$. Denote this set of variables (for all $T$ and $r$ ) by $Y$. Extend the action of the operators $D_{T}$ and $\mathcal{D}_{T}$ to $\mathbb{C}[[z]][Y]$ by making each indeterminate of $Y$ a constant.

Lemma 2.8. Let $r, m \geq 1$ be integers and $S \in \mathbb{T}$. Then

$$
\begin{aligned}
& \sum_{\substack{\left(T_{1}, \ldots, T_{r}\right) \in \mathbb{T}^{r} \\
+\cdots+v\left(T_{r}\right)+v(S)=m}}\left[Y_{T_{1}}^{(1)} \mathcal{D}_{T_{1}}\right] \cdots\left[Y_{T_{r}}^{(r)} \mathcal{D}_{T_{r}}\right] \mathcal{P}_{S}
\end{aligned}
$$

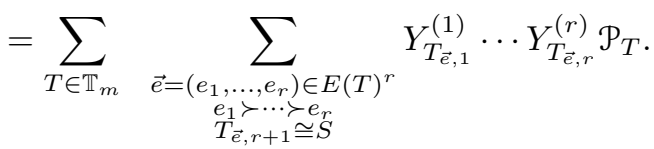


Proof. Note that both sums are finite. So the expression makes sense for any $H \in \mathbb{C}[[z]]^{n}$.

We first consider the case $r=1$. For $T^{\prime} \in \mathbb{T}$ we have

$$
D_{T^{\prime}} P_{S}=\sum_{v \in V(S)} P_{\left(T^{\prime} \multimap_{v} S\right)}
$$

Hence

$$
\begin{aligned}
\sum_{\substack{T^{\prime} \in \mathbb{T} \\
v\left(T^{\prime}\right)+v(S)=m}} Y_{T^{\prime}}^{(1)} D_{T^{\prime}} P_{S} & =\sum_{\substack{T^{\prime} \in \mathbb{T} \\
v\left(T^{\prime}\right)+v(S)=m}} \sum_{v \in V(S)} Y_{T^{\prime}}^{(1)} P_{\left(T^{\prime}-\multimap_{v} S\right)} \\
& =\sum_{T \in \mathbb{T}_{m}} \sum_{T^{\prime} \in \mathbb{T}} \sum_{\substack{v \in V(S) \\
\left(T^{\prime}-o_{v} S\right) \cong T}} Y_{T^{\prime}}^{(1)} P_{\left(T^{\prime}-_{v} S\right)} .
\end{aligned}
$$

For a fixed $T \in \mathbb{T}_{m}$ we wish to count the occurrences of $P_{T}$ in the last expression. Toward this end, for $T^{\prime} \in \mathbb{T}$, let

$$
\begin{aligned}
& I_{T, T^{\prime}, S}=\left\{v \in V(S) \mid\left(T^{\prime} \multimap_{v} S\right) \cong T\right\}, \\
& \left.J_{T, T^{\prime}, S}=\left\{\bar{e} \in E(T) / \operatorname{Aut}(T) \mid T_{e}^{\prime} \cong T^{\prime}, T_{e} \cong S \text { (for any } e \text { in } \bar{e}\right)\right\} .
\end{aligned}
$$

We will define a function $\Phi: I_{T, T^{\prime}, S} \rightarrow J_{T, T^{\prime}, S}$ as follows: Given $v \in I_{T, T^{\prime}, S}$, choose an isomorphism $\varphi:\left(T^{\prime} \multimap v\right) \stackrel{\cong}{\longrightarrow} T$, and let $e$ be the image under $\varphi$ of the connection edge in $T^{\prime} \multimap_{v} S$. Letting $\bar{e}$ be the class of $e$ in $E(T) / \operatorname{Aut}(T)$, we clearly have $\bar{e} \in J_{T, T^{\prime}, S}$. To see that $\bar{e}$ is independent of the choice of $\varphi$, suppose $\gamma:\left(T^{\prime} \multimap v S\right) \stackrel{\cong}{\longrightarrow} T$ sends the connection edge to $f \in E(T)$. Then $\gamma \varphi^{-1}(e)=f$, hence $\bar{f}=\bar{e}$ in $E(T) /$ Aut $(T)$. Therefore, we have a well-defined function $\Phi$, which is obviously surjective.

We claim that for $v \in I_{T, T^{\prime}, S}$ the orbit of $v$ under Aut $(S)$ is precisely the fiber of $v$ under $\Phi$. It is clear that if $w \sim v$ by the action of Aut $(S)$, then $\left(T^{\prime} \multimap w\right.$ $S) \cong\left(T^{\prime} \multimap v S\right) \cong T$, with the first isomorphism taking one connection edge to the other, which shows that $w \in I_{T, T^{\prime}, S}$. Choosing appropriate isomorphisms $\left(T^{\prime} \multimap w S\right) \stackrel{\rho}{\longrightarrow}\left(T^{\prime} \multimap_{v} S\right) \stackrel{\varphi}{\longrightarrow} T$, we see that the image $e$ of the connection edge of $T^{\prime} \multimap_{v} S$ under $\varphi$ is also the image of the connection edge of $T^{\prime} \multimap_{w} S$ under $\varphi \rho$, hence $\Phi(w)=\Phi(v)$. Moreover, if $w \in I_{T, T^{\prime}, S}$ is any element for which $\Phi(w)=\Phi(v)$, then we have isomorphisms

$$
\left(T^{\prime} \multimap_{w} S\right) \stackrel{\gamma}{\longrightarrow} T \stackrel{\varphi}{\longleftarrow}\left(T^{\prime} \multimap_{v} S\right)
$$

such that the same $e \in E(T)$ is the image of both connection edges. (This can be achieved after modifying by an automorphism of $T$.) It follows that $\gamma^{-1} \varphi:\left(T^{\prime} \multimap v\right.$ $S) \rightarrow\left(T^{\prime} \multimap w S\right)$ carries one connection edge to the other; so it restricts to an automorphism of $S$ sending $v$ to $w$. Hence $w \sim v$. Therefore, the above sum can be written as

$$
\begin{aligned}
& \sum_{T \in \mathbb{T}_{m}} \sum_{T^{\prime} \in \mathbb{T}} \sum_{v \in I_{T, T^{\prime}, S}} Y_{T^{\prime}}^{(1)} P_{T} \\
& =\sum_{T \in \mathbb{T}_{m}} \sum_{T^{\prime} \in \mathbb{T}} \sum_{\bar{e} \in J_{T, T^{\prime}, S}} s_{T_{e}}\left(v_{e}\right) Y_{T^{\prime}}^{(1)} P_{T}
\end{aligned}
$$




$$
=\sum_{T \in \mathbb{T}_{m}} \sum_{\substack{\bar{e} \in E(T) / \operatorname{Aut}(T) \\ T_{e} \cong S}} s_{T_{e}}\left(v_{e}\right) Y_{T_{e}^{\prime}}^{(1)} P_{T}
$$

where $s_{T_{e}}\left(v_{e}\right)$ is the orbit size of $v_{e}$ under the action of Aut $T_{e}$, for some (any) $e \in E(T)$ representing $\bar{e}$. The number of edges representing $\bar{e}$ is $\alpha_{T} / \alpha_{T, e}$. Hence the inner sum can be altered to run over all $e \in E(T)$ at the cost of dividing by $\alpha_{T} / \alpha_{T, e}$, yielding

$$
\sum_{T \in \mathbb{T}_{m}} \frac{1}{\alpha_{T}} \sum_{\substack{e \in E(T) \\ T_{e} \cong S}} \alpha_{T, e} s_{T_{e}}\left(v_{e}\right) Y_{T_{e}^{\prime}}^{(1)} P_{T} .
$$

An automorphism of $T$ fixing $e \in E(T)$ restricts to an automorphism of $T_{e}^{\prime}$ and an automorphism of $T_{e}$ fixing $v_{e}$. Conversely, given the latter pair we get a unique automorphism of $T$ preserving $e$. It follows that $\alpha_{T, e}=\alpha_{T_{e}^{\prime}} \alpha_{T_{e}, v_{e}}$. Also we have $s_{T_{e}, v_{e}}=\alpha_{T_{e}} / \alpha_{T_{e}, v_{e}}$. Incorporating these facts and putting together the above equalities, we get

$$
\sum_{\substack{T^{\prime} \in \mathbb{T} \\ v\left(T^{\prime}\right)+v(S)=m}} Y_{T^{\prime}}^{(1)} D_{T^{\prime}} P_{S}=\sum_{T \in \mathbb{T}_{m}} \frac{1}{\alpha_{T}} \sum_{\substack{e \in E(T) \\ T_{e} \cong S}} \alpha_{T_{e}^{\prime}} \alpha_{S} Y_{T_{e}^{\prime}}^{(1)} P_{T} .
$$

Dividing the equation by $\alpha_{S}$ and substituting $\frac{1}{\alpha_{R}} Y_{R}^{(1)}$ for $Y_{R}^{(1)}$ for each $R \in \mathbb{T}$ yields

$$
\sum_{\substack{T^{\prime} \in \mathbb{T} \\ v\left(T^{\prime}\right)+v(S)=m}} Y_{T^{\prime}}^{(1)} \mathcal{D}_{T^{\prime}} \mathcal{P}_{S}=\sum_{T \in \mathbb{T}_{m}} \sum_{\substack{e \in E(T) \\ T_{e} \cong S}} Y_{T_{e}^{\prime}}^{(1)} \mathcal{P}_{T},
$$

which is precisely the assertion of the lemma for $r=1$.

For $r \geq 2$ we apply induction as follows:

$$
\begin{aligned}
& \sum_{\substack{\left(T_{1}, \ldots, T_{r}\right) \in \mathbb{T}^{r} \\
v\left(T_{1}\right)+\cdots+v\left(T_{r}\right)+v(S)=m}}\left[Y_{T_{1}}^{(1)} \mathcal{D}_{T_{1}}\right] \cdots\left[Y_{T_{r}}^{(r)} \mathcal{D}_{T_{r}}\right] \mathcal{P}_{S} \\
& =\sum_{T_{1} \in \mathbb{T}} Y_{T_{1}}^{(1)} \mathcal{D}_{T_{1}} \sum_{\substack{\left(T_{2}, \ldots, T_{r}\right) \in \mathbb{T}^{r-1} \\
v\left(T_{2}\right)+\cdots+v\left(T_{r}\right)+v(S)=m-v\left(T_{1}\right)}}\left[Y_{T_{2}}^{(2)} \mathcal{D}_{T_{2}}\right] \cdots\left[Y_{T_{r}}^{(r)} \mathcal{D}_{T_{r}}\right] \mathcal{P}_{S} .
\end{aligned}
$$

Applying induction and a substitution of variables $Y_{t}^{(i+1)}$ for $Y_{t}^{(i)}$ to the inner sum, this equals

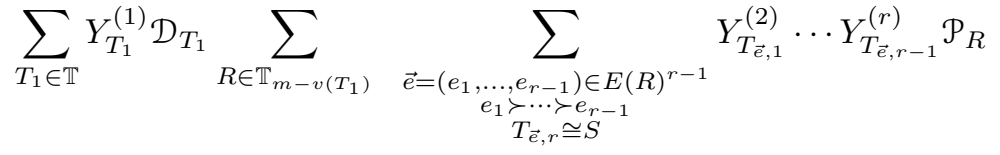

$$
\begin{aligned}
& =\sum_{\substack{T_{1}, R \in \mathbb{T} \\
v\left(T_{1}\right)+v(R)=m}} Y_{T_{1}}^{(1)} \mathcal{D}_{T_{1}} \sum_{\substack{\vec{e}=\left(e_{1}, \ldots, e_{r-1}\right) \in E(R)^{r-1} \\
e_{1} \succ \cdots \succ e_{r-1} \\
T_{\vec{e}, r} \cong S}} Y_{T_{\vec{e}, 1}}^{(2)} \cdots Y_{T_{\vec{e}, r-1}}^{(r)} \mathcal{P}_{R}
\end{aligned}
$$

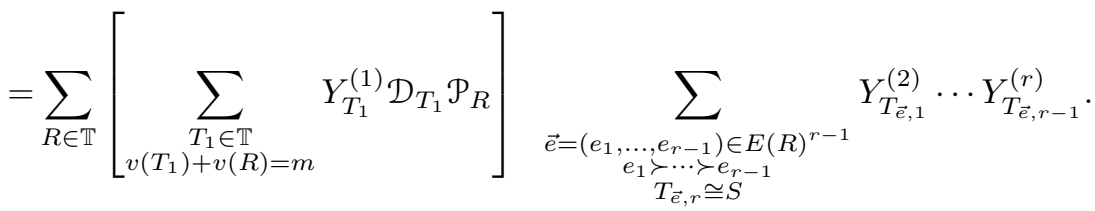


Now we apply the case $r=1$ to the bracketed expression to obtain

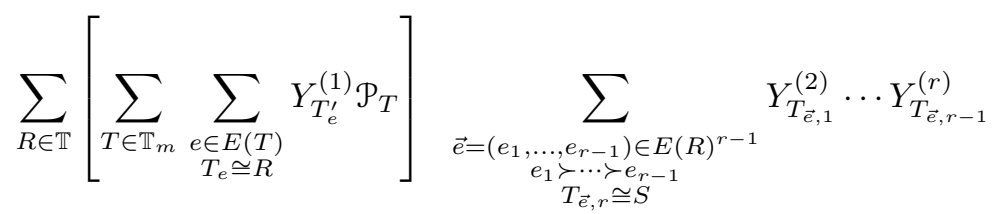

$$
\begin{aligned}
& \cdot \sum_{T \in \mathbb{T}_{m}} \sum_{R \in \mathbb{T}} \sum_{\substack{e \in E(T) \\
T_{e} \cong R}} \sum_{\substack{\vec{e}=\left(e_{1}, \ldots, e_{r-1}\right) \in E(R)^{r-1} \\
e_{e}, \ldots \succ e_{r}-1 \\
T_{\vec{e}, r} \cong S}} Y_{T_{e}^{\prime}}^{(1)} Y_{T_{\vec{e}, 1}}^{(2)} \cdots Y_{T_{\vec{e}, r-1}}^{(r)} \mathcal{P}_{T}
\end{aligned}
$$

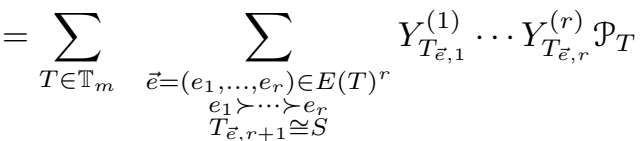

which completes the proof.

Suppose the system of power series $H=\left(H_{1}, \ldots, H_{n}\right)$ has the property that each $H_{i}$ involves only monomials of degree $\geq 2$ in $z_{1}, \ldots, z_{n}$. Then one easily verifies that for $T \in \mathbb{T}, \mathcal{P}_{T}$ involves only monomials of degree $\geq v(T)+1$. It follows that for a monomial $M$ in $z$ of degree $m, \mathcal{D}_{T} \cdot M$ involves only monomials of degree $\geq m+v(T)$. Therefore, infinite sums such as $\sum_{T \in \mathbb{T}} \mathcal{P}_{T}$ and $\sum_{T \in \mathbb{T}} \mathcal{D}_{T}$ make sense in this situation. The following two corollaries of Lemma 2.8 are based on this observation. The equations in both corollaries take place in the ring $\mathbb{C}[Y][[z]]$, where $Y$ represents the infinite set of variables $\left\{Y_{T}^{(i)} \mid T \in \mathbb{T}, i \in \mathbb{Z}^{+}\right\}$.

Corollary 2.9. Suppose that the system of power series $H$ involves only monomials of degree $\geq 2$. Let $r \geq 1$ be an integer and $S \in \mathbb{T}$. Then

$$
\begin{aligned}
\sum_{\left(T_{1}, \ldots, T_{r}\right) \in \mathbb{T}^{r}}\left[Y_{T_{1}}^{(1)} \mathcal{D}_{T_{1}}\right] \cdots\left[Y_{T_{r}}^{(r)} \mathcal{D}_{T_{r}}\right] \mathcal{P}_{S} & \sum_{T \in \mathbb{T}} \sum_{\substack{\vec{e}=\left(e_{1}, \ldots, e_{r}\right) \in E(T)^{r} \\
e_{1} \succ, .<e_{r} \\
T_{\vec{e}, r+1}=S}} Y_{T_{\vec{e}, 1}}^{(1)} \cdots Y_{T_{\vec{e}, r}}^{(r)} \mathcal{P}_{T} .
\end{aligned}
$$

Proof. We simply sum (2.1) over all $m \geq 1$, noting the convergence of the sums by the observations above.

Corollary 2.10. Suppose the system of power series $H$ involves only monomials of degree $\geq 2$. Let $k \geq 2$ be an integer. Then

$$
\begin{aligned}
\sum_{\left(T_{1}, \ldots, T_{k}\right) \in \mathbb{T}^{k}}\left[Y_{T_{1}}^{(1)} \mathcal{D}_{T_{1}}\right] \cdots\left[Y_{T_{k-1}}^{(k-1)} \mathcal{D}_{T_{k-1}}\right]\left[Y_{T_{k}}^{(k)} \mathcal{P}_{T_{k}}\right] & \sum_{\substack{T \in \mathbb{T} \\
v(T) \geq 2}} \sum_{\substack{\vec{e}=\left(e_{1}, \ldots, e_{k-1}\right) \in E(T)^{k-1} \\
e_{1} \succ \cdots \succ e_{k-1}}} Y_{T_{\vec{e}, 1}}^{(1)} \cdots Y_{T_{\vec{e}, k}}^{(k)} \mathcal{P}_{T} .
\end{aligned}
$$

Proof. We apply Corollary 2.9. multiplying both sides of (2.2) by $Y_{S}^{(r+1)}$, setting $k=r+1$, summing over all $S \in \mathbb{T}$. Note that the singleton tree contributes 0 in (2.2) for any $r \geq 1$, and thus the qualifier $v(T) \geq 2$ in (2.3). 


\section{D-Log And Formal Flow}

We will henceforth be restricting our attention to systems of power series $F=$ $\left(F_{1}, \ldots, F_{n}\right) \in \mathbb{C}[[z]]^{n}$ of the form $F_{i}=z_{i}+H_{i}$ with $H_{i}$ involving only monomials of degree 2 and higher, for $i=1, \ldots, n$. We refer to this condition by saying " $F$ is of the form identity plus higher." Such a system determines a $\mathbb{C}$-algebra automorphism of $\mathbb{C}[[z]]$, namely the automorphism that sends $z_{i}$ to $F_{i}$ for $i=1, \ldots, n$.

3.1. The D-Log. The following proposition appears as Proposition 2.1 in $[\mathrm{Z}]$.

Proposition 3.1. For any $F=\left(F_{1}, F_{2}, \ldots, F_{n}\right) \in \mathbb{C}[[z]]^{n}$ of the form identity plus higher, there exists a unique system of power series

$$
a=\left(a_{1}, a_{2}, \ldots, a_{n}\right) \in \mathbb{C}[[z]]^{n}
$$

involving only monomials of degree 2 and higher such that, letting $A=a D=$ $\sum_{i=1}^{n} a_{i} D_{i}$, we have

$$
\exp (A) \cdot z=F
$$

where

$$
\exp (A)=\sum_{k=0}^{\infty} \frac{A^{k}}{k !}
$$

and $z=\left(z_{1}, \ldots, z_{n}\right)$.

The reader will easily verify that the infinite $\operatorname{sum} \exp (A) \cdot Q$ makes sense for any $Q \in \mathbb{C}[[z]]^{n}$ due to the fact that, for any integer $d \geq 0$, only finitely many terms $\frac{A^{k}}{k !} \cdot Q$ contribute to the degree $d$ homogeneous summand. This is due to the fact that $a$ involves only terms of degree 2 and higher.

Remark 3.2. It is well known that the exponential of a derivation on any $\mathbb{Q}$-algebra, when it makes sense, is an automorphism of that algebra. Any subring lying in the kernel of the derivation will be fixed by this automorphism. It follows from this fact, the comment above, and Proposition 3.1 that $\exp (A)$ is the $\mathbb{C}$-algebra automorphism of $\mathbb{C}[[z]]$ that sends $z_{i}$ to $F_{i}$, for $i=1, \ldots, n$.

Definition 3.3. We call the unique system of power series $a=\left(a_{1}, a_{2}, \ldots, a_{n}\right)$ obtained above the Differential $\log$ or $D$ - $\log$ of the formal system $F$.

\subsection{Coefficients $\phi_{T}$ of the D-Log.}

Theorem 3.4. There exists a unique set of rational numbers $\left\{\phi_{T}\right\}$ indexed by the set of rooted trees $T \in \mathbb{T}$, such that

$$
a=\sum_{T \in \mathbb{T}} \phi_{T} \mathcal{P}_{T}
$$

These numbers satisfy, and are uniquely determined by, the following properties:

$$
\begin{aligned}
& \phi_{T}=1 \text { when } v(T)=1 \text { (i.e., } T=\circ \text {, the singleton tree), } \\
& \phi_{T}=-\sum_{k=2}^{v(T)} \frac{1}{k !} \sum_{\substack{\vec{e}=\left(e_{1}, \ldots, e_{k-1}\right) \in E(T)^{k-1} \\
e_{1} \succ \cdots \succ e_{k-1}}} \phi_{T_{\vec{e}, 1}} \phi_{T_{\vec{e}, 2}} \cdots \phi_{T_{\vec{e}, k}} \text { when } v(T) \geq 2 .
\end{aligned}
$$


The latter formula can be restated as

$$
\sum_{k=1}^{v(T)} \frac{1}{k !} \sum_{\substack{\vec{e}=\left(e_{1}, \ldots, e_{k-1}\right) \in E(T)^{k-1} \\ e_{1} \succ \cdots \succ e_{k-1}}} \phi_{T_{\vec{e}, 1}} \phi_{T_{\vec{e}, 2}} \cdots \phi_{T_{\vec{e}, k}}=0 .
$$

(Here we must interpret the $k=1$ summand as $\phi_{T}$.)

Proof. Let us define $\phi_{T}$ by (3.3) and set $a^{\prime}=\sum_{T \in \mathbb{T}} \phi_{T} \mathcal{P}_{T}, A^{\prime}=a^{\prime} D$. Then $A^{\prime}=\sum_{T \in \mathbb{T}} \phi_{T} \mathcal{D}_{T}$. We have

$$
\begin{aligned}
\exp \left(A^{\prime}\right) & \cdot z=\sum_{k=0}^{\infty} \frac{A^{\prime k}}{k !} \cdot z \\
& =\sum_{k=0}^{\infty} \frac{1}{k !}\left(\sum_{T \in \mathbb{T}} \phi_{T} \mathcal{D}_{T}\right)^{k} \cdot z \\
& =z+\sum_{k=1}^{\infty} \frac{1}{k !} \sum_{\left(T_{1}, \ldots, T_{k}\right) \in \mathbb{T}^{k}}\left[\phi_{T_{1}} \mathcal{D}_{T_{1}}\right] \cdots\left[\phi_{T_{k}} \mathcal{D}_{T_{k}}\right] \cdot z \\
& =z+\sum_{T \in \mathbb{T}} \phi_{T} \mathcal{D}_{T} \cdot z+\sum_{k=2}^{\infty} \frac{1}{k !} \sum_{\left(T_{1}, \ldots, T_{k}\right) \in \mathbb{T}^{k}}\left[\phi_{T_{1}} \mathcal{D}_{T_{1}}\right] \cdots\left[\phi_{T_{k}} \mathcal{D}_{T_{k}}\right] \cdot z
\end{aligned}
$$

Using the fact that $\mathcal{D}_{T} \cdot z=\mathcal{P}_{T}$ :

$$
=z+\sum_{T \in \mathbb{T}} \phi_{T} \mathcal{P}+\sum_{k=2}^{\infty} \frac{1}{k !} \sum_{\left(T_{1}, \ldots, T_{k}\right) \in \mathbb{T}^{k}}\left[\phi_{T_{1}} \mathcal{D}_{T_{1}}\right] \cdots\left[\phi_{T_{k-1}} \mathcal{D}_{T_{k-1}}\right]\left[\phi_{T_{k}} \mathcal{P}_{T_{k}}\right]
$$

Applying Corollary 2.10 substituting $Y_{T_{i}}^{(i)}=\phi_{T_{i}}$ :

$$
=z+\sum_{T \in \mathbb{T}} \phi_{T} \mathcal{P}_{T}+\sum_{k=2}^{\infty} \frac{1}{k !} \sum_{\substack{T \in \mathbb{T} \\ v(T) \geq 2}} \sum_{\substack{\vec{e}=\left(e_{1}, \ldots, e_{k-1}\right) \in E(T)^{k-1} \\ e_{1} \succ \cdots \succ e_{k-1}}} \phi_{T_{\vec{e}, 1}} \cdots \phi_{T_{\vec{e}, k}} \mathcal{P}_{T} .
$$

Letting $S$ be the singleton tree:

$$
=z+\phi_{S} \mathcal{P}_{S}+\sum_{\substack{T \in \mathbb{T} \\ v(T) \geq 2}}\left(\sum_{k=1}^{v(T)} \frac{1}{k !} \sum_{\substack{\vec{e}=\left(e_{1}, \ldots, e_{k-1}\right) \in E(T)^{k-1} \\ e_{1} \succ \cdots \succ e_{k-1}}} \phi_{T_{\vec{e}, 1}} \cdots \phi_{T_{\vec{e}, k}}\right) \mathcal{P}_{T} .
$$

Since $\mathcal{P}_{S}=H$, and, by definition, $\phi_{S}=1$ and the sum in parentheses is 0 :

$$
=z+H=F \text {. }
$$

By the uniqueness property of $a$ we must have $a^{\prime}=a$. The uniqueness of the expression (3.2) for $a$ follows from Theorem 2.4.

Chains and Shrubs. Two special types of trees are the "chains" and the "shrubs", mentioned in $\S 2$. Given an integer $n \geq 1$ we let $C_{n} \in \mathbb{T}_{n}$ be the chain with $n$ vertices, which is the unique rooted tree in $\mathbb{T}_{n}$ of height $n-1$. For $n \geq 0$ we let $S_{n} \in \mathbb{T}_{n+1}$ be the shrub with $n+1$ vertices, which is the unique rooted tree in $\mathbb{T}_{n+1}$ 
of height $\leq 1$ (equality holds unless $n=0$ ). Note that $C_{1}=S_{0}=\circ$, the singleton tree.

By using the recurrence formula (3.3), we can calculate $\phi_{T}$ for chains and shrubs as follows. Consider the generating functions

$$
\begin{aligned}
& c(x)=\sum_{n=1}^{\infty} \phi_{C_{n}} x^{n}, \\
& s(x)=\sum_{n=0}^{\infty} \phi_{S_{n}} \frac{x^{n}}{n !} .
\end{aligned}
$$

Then we have:

Corollary 3.5. The generating functions $c(x)$ and $s(x)$ are given by

$$
\begin{aligned}
& \text { (a) } \quad c(x)=\ln (1+x), \\
& \text { (b) } \quad s(x)=\frac{x}{e^{x}-1} .
\end{aligned}
$$

In particular, we have $\phi_{C_{n}}=(-1)^{n-1} \frac{1}{n}$ for all $n \geq 1$ and $\phi_{S_{n}}=b_{n}$, where $b_{0}, b_{1}, b_{2}, \ldots$ are the Bernoulli numbers 3 defined by $\frac{x}{e^{x}-1}=\sum_{n=0}^{\infty} b_{n} \frac{x^{n}}{n !}$.

Proof. (a) According to (3.3) we have

$$
c(x)=x-\sum_{n=2}^{\infty}\left(\sum_{k=2}^{v\left(C_{n}\right)} \frac{1}{k !} \sum_{\substack{\vec{e}=\left(e_{1}, \ldots, e_{k-1}\right) \in E\left(C_{n}\right)^{k-1} \\ e_{1} \succ \cdots \succ e_{k-1}}} \phi_{T_{\vec{e}, 1}} \phi_{T_{\vec{e}, 2}} \cdots \phi_{T_{\vec{e}, k}}\right) x^{n} .
$$

Noting that $v\left(C_{n}\right)=n$ and each $T_{\vec{e}, j}$ is also a path:

$$
\begin{aligned}
& =x-\sum_{n=2}^{\infty} \sum_{k=2}^{n} \frac{1}{k !} \sum_{\substack{\left(i_{1}, \ldots, i_{k}\right) \in \mathbb{N}^{k} \\
i_{1}+\cdots+i_{k}=n}} \prod_{j=1}^{k} \phi_{C_{i_{j}}} x^{i_{j}} \\
& =x-\sum_{n=2}^{\infty}\left(\sum_{k=2}^{n} \frac{1}{k !}\left(\text { coefficient of } x^{n} \text { in } c(x)^{k}\right)\right) x^{n} \\
& =x-\sum_{n=2}^{\infty}\left(\text { coefficient of } x^{n} \text { in } \sum_{k=2}^{n} \frac{1}{k !} c(x)^{k}\right) x^{n} \\
& =x-\sum_{n=2}^{\infty}\left(\text { coefficient of } x^{n} \text { in } \sum_{k=2}^{\infty} \frac{1}{k !} c(x)^{k}\right) x^{n} \\
& =x-\sum_{n=2}^{\infty} \frac{1}{n !} c(x)^{n} \\
& =x-\left(e^{c(x)}-c(x)-1\right) .
\end{aligned}
$$

\footnotetext{
${ }^{3}$ This indexing and signage differs from an alternate definition of the Bernoulli numbers as the sequence $B_{1}, B_{2}, \ldots$ defined by

$$
\frac{x}{e^{x}-1}=1-\frac{1}{2} x+\sum_{n=1}^{\infty}(-1)^{n-1} \frac{B_{n}}{(2 n) !} x^{2 n}
$$

Thus the relationship is $B_{n}=(-1)^{n-1} b_{2 n}$ for $n \geq 1$.
} 
Solving for $c(x)$ in the equation $c(x)=x-\left(e^{c(x)}-c(x)-1\right)$ gives (3.5).

(b) Again by (3.3) we have

$$
s(x)=1-\sum_{n=1}^{\infty}\left(\sum_{k=2}^{v\left(S_{n}\right)} \frac{1}{k} \sum_{\substack{\vec{e}=\left(e_{1}, \ldots, e_{k-1}\right) \in E\left(S_{n}\right)^{k-1} \\ e_{1} \succ \cdots \succ e_{k-1}}} \phi_{T_{\vec{e}, 1}} \phi_{T_{\vec{e}, 2}} \cdots \phi_{T_{\vec{e}, k}}\right) \frac{x^{n}}{n !} .
$$

Noting that $v\left(S_{n}\right)=n+1$ and precisely one $T_{\vec{e}, j}$ is a shrub with all others being singletons:

$$
\begin{aligned}
& =1-\sum_{n=1}^{\infty}\left(\sum_{k=2}^{n+1} \frac{1}{k !}(k-1) !\left(\begin{array}{c}
n \\
k-1
\end{array}\right) \phi_{S_{n-k+1}}\right) \frac{x^{n}}{n !} \\
& =1-x^{-1} \sum_{n=1}^{\infty} \sum_{k=2}^{n+1} \phi_{S_{n-k+1}} \frac{x^{n-k+1}}{(n-k+1) !} \frac{x^{k}}{k !} \\
& =1-x^{-1}\left(\sum_{r=0}^{\infty} \phi_{S_{r}} \frac{x^{r}}{r !}\right)\left(\sum_{s=2}^{\infty} \frac{x^{s}}{s !}\right) \\
& =1-x^{-1} s(x)\left(e^{x}-x-1\right) .
\end{aligned}
$$

Solving for $s(x)$ in the equation $s(x)=1-x^{-1} s(x)\left(e^{x}-x-1\right)$ gives (3.6).

3.3. Polynomial Coefficients $\psi_{T}(t)$ of Formal Flow. Let us first recall the formal flow $F_{t}=\exp (t A) \cdot z$ and some of its properties. See [Z] for more details.

Definition 3.6. Given an indeterminate $t$, define the system $F_{t} \in \mathbb{C}[t][[z]]^{n}$ by

$$
F_{t}=\exp (t A) \cdot z .
$$

It is called the formal flow generated by $F$.

It is easy to verify that $F_{t} \in \mathbb{C}[t][[z]]^{n}$. Therefore, a specialization $t=\alpha$, for any $\alpha \in \mathbb{C}$ (or $\alpha$ in any $\mathbb{C}$-algebra), makes sense. According to Proposition (3.1), setting $t=1$ in $F_{t}$ recovers $F$.

The following proposition shows that $t$ behaves as an exponent for $F$.

Proposition 3.7. Let $t$ and $s$ be indeterminates. Then

$$
F_{s+t}=F_{t} \circ F_{s} .
$$

Hence setting $t=n$ in $F_{t}$, for $n \in \mathbb{N}$, gives the $n$-fold composition $F \circ \cdots \circ F$, and setting $t=-n$ gives the $n$-fold composition $F^{-1} \circ \cdots \circ F^{-1}$ of the formal inverse. In particular,

$$
\left.F_{t}\right|_{t=-1}=F^{-1} .
$$

Proof. We have

$$
\begin{aligned}
F_{s+t} & =\exp ((s+t) A) \cdot z=\exp (s A+t A) \cdot z \\
& =\exp (s A) \cdot \exp (t A) \cdot z=\exp (s A) \cdot F_{t} .
\end{aligned}
$$

We use the fact that $\exp (s A)$ is a $\mathbb{C}$-algebra automorphism of $\mathbb{C}[s, t][[z]]$ (see Remark 3.2):

$$
\begin{aligned}
& =F_{t}(\exp (s A \cdot z))=F_{t}\left(F_{s}\right) \\
& =F_{t} \circ F_{s} .
\end{aligned}
$$


Thus $F_{t}$ can be viewed as the "formal $t$-th power of $F$ ".

The system $F_{t}$ can be expressed in terms of the tree expressions $\mathcal{P}_{T}$ as follows:

Theorem 3.8. There exists a unique set of polynomials $\left\{\psi_{T}(t) \in \mathbb{Q}[t]\right\}$ indexed by the set of rooted trees $T \in \mathbb{T}$ such that

$$
F_{t}=z+\sum_{T \in \mathbb{T}} \psi_{T}(t) \mathcal{P}_{T} .
$$

These polynomials are given by the formula

$$
\psi_{T}(t)=\sum_{k=1}^{v(T)} \frac{t^{k}}{k !} \sum_{\substack{\vec{e}=\left(e_{1}, \ldots, e_{k-1}\right) \in E(T)^{k-1} \\ e_{1} \succ \cdots \succ e_{k-1}}} \phi_{T_{\vec{e}, 1}} \phi_{T_{\vec{e}, 2}} \cdots \phi_{T_{\vec{e}, k}} .
$$

(Again we must interpret the $k=1$ summand as $\phi_{T}$.)

Proof. According to Theorem 3.4 the D-Log of $F$ is given by $a=\sum_{T \in \mathbb{T}} \phi_{t} \mathcal{P}_{T}$. Hence we have $A=a D=\sum_{T \in \mathbb{T}} \phi_{t} \mathcal{P}_{T} D=\sum_{T \in \mathbb{T}} \phi_{T} \mathcal{D}_{T}$ (see Definition 2.7). Therefore,

$$
\begin{aligned}
F_{t} & =\exp (t A) \cdot z=\sum_{k=0}^{\infty} \frac{t^{k}}{k !} A^{k} \cdot z \\
& =z+\sum_{k=1}^{\infty} \frac{t^{k}}{k !}\left(\sum_{T \in \mathbb{T}} \phi_{T} \mathcal{D}_{T}\right)^{k} \cdot z \\
& =z+\sum_{k=1}^{\infty} \frac{t^{k}}{k !} \sum_{\left(T_{1}, \ldots, T_{k}\right) \in \mathbb{T}}\left[\phi_{T_{1}} \mathcal{D}_{T_{1}}\right] \cdots\left[\phi_{T_{k}} \mathcal{D}_{T_{k}}\right] \cdot z \\
& =z+\sum_{k=1}^{\infty} \frac{t^{k}}{k !} \sum_{\left(T_{1}, \ldots, T_{k}\right) \in \mathbb{T}}\left[\phi_{T_{1}} \mathcal{D}_{T_{1}}\right] \cdots\left[\phi_{T_{k-1}} \mathcal{D}_{T_{k-1}}\right]\left[\phi_{T_{k}} \mathcal{P}_{T_{k}}\right] .
\end{aligned}
$$

Now we apply Corollary 2.10 to the $k \geq 2$ summands:

$$
\begin{aligned}
& =z+\sum_{k=1}^{\infty} \frac{t^{k}}{k !} \sum_{T \in \mathbb{T}} \sum_{\substack{\vec{e}=\left(e_{1}, \ldots, e_{k-1) \in E(T)^{k-1}} e_{1} \succ \cdots \succ e_{k-1}\\
\\
\right.}} \phi_{T_{\vec{e}, 1}} \cdots \phi_{T_{\vec{e}, k}} \mathcal{P}_{T} \\
& =z+\sum_{T \in \mathbb{T}}\left(\sum_{k=1}^{v(T)} \frac{t^{k}}{k !} \sum_{\substack{\vec{e}=\left(e_{1}, \ldots, e_{k-1}\right) \in E(T)^{k-1} \\
e_{1} \succ \cdots \succ e_{k-1}}} \phi_{T_{\vec{e}, 1}} \cdots \phi_{T_{\vec{e}, k}}\right) \mathcal{P}_{T} .
\end{aligned}
$$

This gives the desired result. The uniqueness of $\psi_{T}$ follows from applying stable linear independence (Corollary 2.6) to each power of $t$ in (3.10).

Lemma 3.9. For any $T \in \mathbb{T}$, we have

(1) if $T$ is the singleton, we have $\psi_{T}(t)=t$.

(2) $\psi_{T}(0)=0$.

(3) $\psi_{T}(1)= \begin{cases}1 & \text { if } v(T)=1, \\ 0 & \text { if } v(T) \geq 2 .\end{cases}$

(4) $\psi_{T}^{\prime}(0)=\phi_{T}$. 
Proof. All statements above follow immediately from (3.11), except the assertion $\psi_{T}(1)=0$ when $v(T) \geq 2$, which is exactly (3.4).

Forests. The formula (3.11) defines a unique polynomial $\psi_{T}(t)$ for each rooted tree $T$. A forest is the disjoint union of finitely many rooted trees. We extend the definitions of $\phi_{P}$ and $\psi_{P}(t)$ to any forest $P$ as follows:

Definition 3.10. For any forest $P$ that is the disjoint union of rooted trees $T_{1}, \ldots, T_{k}$, we define $\phi_{P}$ to be $\phi_{T_{1}}$ if $k=1$ and 0 otherwise. Define $\psi_{P}(t)=$ $\prod_{i=1}^{k} \psi_{T_{i}}(t)$.

Lemma 3.11. Let $T$ be a rooted tree with $v(T) \geq 2$. For any proper rooted subtree $T^{\prime}$ of $T$ we have

$$
\psi_{T \backslash T^{\prime}}(t)=\sum_{k=1}^{v(T)-1} \frac{t^{k}}{k !} \sum_{\substack{\vec{e}=\left(e_{1}, \ldots, e_{k}\right) \in E(T)^{k} \\ e_{1} \succ \ldots \succ e_{k} \\ T_{\vec{e}, k+1}=T^{\prime}}} \phi_{T_{\vec{e}, 1}} \phi_{T_{\vec{e}, 2}} \cdots \phi_{T_{\vec{e}, k}} .
$$

Proof. Let $T^{[j]}(j=1,2, \ldots, d)$ be the connected components of $T \backslash T^{\prime}$, and let $e_{j}^{0}$ be the edge of $T$ that connects $T^{[j]}$ with $T^{\prime}$. Note that from fixed sequences $e_{j, 1} \succ e_{j, 2} \succ \cdots \succ e_{j, k_{j}} \in E\left(T^{[j]}\right)$ with $k_{1}+k_{2}+\cdots+k_{d}=k-d$, appended by the edges $e_{j}^{0}$, we can get $\left(\begin{array}{c}k \\ \left(k_{1}+1\right),\left(k_{2}+1\right), \cdots,\left(k_{d}+1\right)\end{array}\right)=\frac{k !}{\left(k_{1}+1\right) !\left(k_{2}+1\right) ! \cdots\left(k_{d}+1\right) !}$ different sequences $e_{1} \succ e_{2} \succ \cdots \succ e_{k} \in E(T)$ such that $T_{k+1}=T^{\prime}$. Therefore,

$$
\begin{aligned}
& \sum_{k=1}^{v(T)-1} \frac{t^{k}}{k !} \sum_{\substack{\vec{e}=\left(e_{1}, \ldots, e_{k}\right) \in E(T)^{k} \\
e_{1} \succ \ldots \\
T_{\vec{e}, k+1}=e_{k}, T^{\prime}}} \phi_{T_{\vec{e}, 1}} \phi_{T_{\vec{e}, 2}} \cdots \phi_{T_{\vec{e}, k}} \\
& =\sum_{k=1}^{v(T)-1} \frac{t^{k}}{k !} \sum_{\substack{\left(k_{1}, \ldots, k_{d}\right) \in \mathbb{N}^{d} \\
k_{1}+k_{2}+\cdots+k_{d}=k-d}} \frac{k !}{\left(k_{1}+1\right) !\left(k_{2}+1\right) ! \cdots\left(k_{d}+1\right) !} \\
& \cdot \prod_{j=1}^{d} \sum_{\substack{\vec{e}_{j}=\left(e_{j, 1}, \ldots, e_{j, k_{j}}\right) \in E\left(T^{[j]}\right)^{k_{j}} \\
e_{j, 1} \succ \cdots \succ e_{j, k_{j}}}} \phi_{T_{e_{j}, 1}} \phi_{T_{e_{j}, 2}} \cdots \phi_{T_{e_{j}, k_{j}+1}} \\
& =\prod_{j=1}^{d} \sum_{k_{j}=0}^{v\left(T^{[j]}\right)-1} \frac{t^{k_{j}+1}}{\left(k_{j}+1\right) !} \sum_{\substack{\vec{e}_{j}=\left(e_{j, 1}, \ldots, e_{j, k_{j}}\right) \in E\left(T^{[j]}\right)^{k_{j}} \\
e_{j, 1} \succ \cdots \succ e_{j, k_{j}}}} \phi_{T_{j, 1}} \phi_{T_{j, 2}} \cdots \phi_{T_{j, k_{j}+1}} \\
& =\psi_{T^{[1]}}(t) \psi_{T^{[2]}}(t) \cdots \psi_{T^{[d]}}(t) \text {. }
\end{aligned}
$$

The last equality follows from (3.11).

The lemma above allows us to prove the following theorem. If we let $\emptyset$ be the empty tree and define $\mathcal{P}_{\emptyset}=z$, then Theorem 3.8 can be seen as the special case $S=\emptyset$ of the theorem below. 
Theorem 3.12. For any rooted tree $S$, we have

$$
\exp (t A) \cdot \mathcal{P}_{S}=\mathcal{P}_{S}+\sum_{T \in \mathbb{T}}\left(\sum_{\substack{T^{\prime}<T \\ T^{\prime} \cong S}} \psi_{T \backslash T^{\prime}}(t)\right) \mathcal{P}_{T} .
$$

Proof.

$$
\begin{aligned}
\exp (t A) \cdot \mathcal{P}_{S} & =\sum_{k=0}^{\infty} \frac{t^{k}}{k !} A^{k} \cdot \mathcal{P}_{S} \\
& =\sum_{k=0}^{\infty} \frac{t^{k}}{k !}\left(\sum_{T \in \mathbb{T}} \phi_{T} \mathcal{D}\right)^{k} \cdot \mathcal{P}_{S} \\
& =\mathcal{P}_{S}+\sum_{k=1}^{\infty} \frac{t^{k}}{k !} \sum_{\left(T_{1}, \ldots, T_{k}\right) \in \mathbb{T}^{k}}\left[\phi_{T_{1}} \mathcal{D}_{T_{1}}\right] \cdots\left[\phi_{T_{k}} \mathcal{D}_{T_{k}}\right] \cdot \mathcal{P}_{S}
\end{aligned}
$$

Apply Corollary 2.9, substituting $Y_{T_{i}}^{(i)}=\phi_{T_{i}}$ :

$$
\begin{aligned}
& =\mathcal{P}_{S}+\sum_{k=1}^{\infty} \frac{t^{k}}{k !} \sum_{\substack{T \in \mathbb{T} \\
v(T) \geq 1}} \sum_{\substack{\vec{e}=\left(e_{1}, \ldots, e_{k-1}\right) \in E(T)^{k} \\
e_{1} \succ \ldots \succ e_{k} \\
T_{\vec{e}, k+1} \cong S}} \phi_{T_{\vec{e}, 1}} \cdots \phi_{T_{\vec{e}, k}} \mathcal{P}_{T}
\end{aligned}
$$

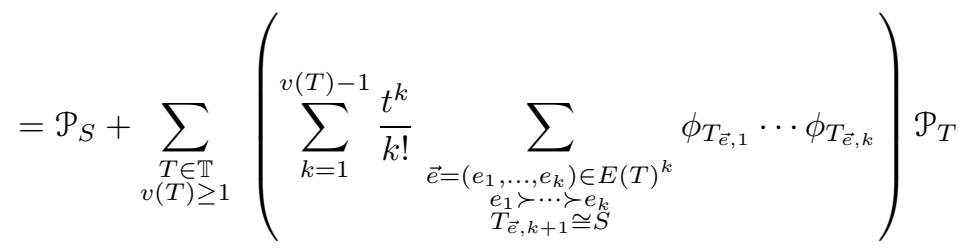

$$
\begin{aligned}
& =\sum_{T \in \mathbb{T}}\left(\sum_{\substack{T^{\prime} \leq T \\
T^{\prime} \cong S}} \psi_{T \backslash T^{\prime}}(t)\right) \mathcal{P}_{T} .
\end{aligned}
$$

The last equality follows from Lemma 3.11.

Proposition 3.13. For any rooted tree $T$, we have

$$
\psi_{T}^{\prime}(t)=\phi_{T}+\sum_{e \in E(T)} \phi_{T_{e, 1}} \psi_{T_{e, 2}}(t),
$$

$$
\psi_{T}^{\prime}(t)=\phi_{T}+\sum_{S<T} \phi_{S} \psi_{T \backslash S}(t)
$$

or, in other words,

$$
\begin{aligned}
\psi_{T}^{\prime}(t) & =\psi_{T}^{\prime}(0)+\sum_{e \in E(T)} \psi_{T_{e, 1}}^{\prime}(0) \psi_{T_{e, 2}}(t) \\
& =\psi_{T}^{\prime}(0)+\sum_{S<T} \psi_{S}^{\prime}(0) \psi_{T \backslash S}(t) .
\end{aligned}
$$


Proof. (a) Applying the chain rule and Theorem 3.8 we have

$$
\begin{aligned}
\frac{\partial}{\partial t} F_{t} & =\frac{\partial}{\partial t}(\exp (t A) \cdot z) \\
& =A \cdot \exp (t A) \cdot z \\
& =\left(\sum_{T \in \mathbb{T}} \phi_{T} \mathcal{D}_{T}\right) \cdot\left(z+\sum_{T \in \mathbb{T}} \psi_{T}(t) \mathcal{P}_{T}\right) \\
& =\sum_{T \in \mathbb{T}} \phi_{T} \mathcal{P}_{T}+\sum_{\left(T_{1}, T_{2}\right) \in \mathbb{T}^{2}} \phi_{T_{1}} \psi_{T_{2}}(t) \mathcal{D}_{T_{1}} \mathcal{P}_{T_{2}} .
\end{aligned}
$$

Applying Corollary[2.10 with $k=2$, setting $Y_{T}^{(1)}=\phi_{T}, Y_{T}^{(2)}=\psi_{T}(t)$ for all $T \in \mathbb{T}$ :

$$
\begin{aligned}
& =\sum_{T \in \mathbb{T}} \phi_{T} \mathcal{P}_{T}+\sum_{T \in \mathbb{T}} \sum_{e \in E(T)} \phi_{T_{e, 1}} \psi_{T_{e, 2}}(t) \mathcal{P}_{T} \\
& =\sum_{T \in \mathbb{T}}\left(\phi_{T}+\sum_{e \in E(T)} \phi_{T_{e, 1}} \psi_{T_{e, 2}}(t)\right) \mathcal{P}_{T} .
\end{aligned}
$$

But we also have, by Theorem 3.8

$$
\frac{\partial}{\partial t} F_{t}=\sum_{T \in \mathbb{T}} \psi_{T}^{\prime}(t) \mathcal{P}_{T}
$$

Comparing the coefficient of $\mathcal{P}_{T}$, and appealing to stable linear independencespecifically, Corollary 2.6 - we get (3.14). (We use the fact that polynomial functions that agree at all $\alpha \in \mathbb{C}$ must be equal.)

(b)

$$
\begin{aligned}
\frac{\partial}{\partial t} F_{t} & =\frac{\partial}{\partial t} \exp (t A) \cdot z \\
& =A \cdot \exp (t A) \cdot z \\
& =\exp (t A) \cdot A \cdot z \\
& =\exp (t A) \cdot a .
\end{aligned}
$$

Applying Theorem 3.4

$$
\begin{aligned}
& =\exp (t A) \cdot \sum_{S \in \mathbb{T}} \phi_{S} \mathcal{P}_{S} \\
& =\sum_{S \in \mathbb{T}} \phi_{S} \exp (t A) \cdot \mathcal{P}_{S} .
\end{aligned}
$$


Applying Theorem 3.12

$$
\begin{aligned}
& =\sum_{S \in \mathbb{T}} \phi_{S} \mathcal{P}_{S}+\sum_{S \in \mathbb{T}} \phi_{S} \sum_{T \in \mathbb{T}}\left(\sum_{\substack{T^{\prime}<T \\
T^{\prime} \cong S}} \psi_{T \backslash T^{\prime}}(t)\right) \mathcal{P}_{T} \\
& =\sum_{T \in \mathbb{T}} \phi_{T} \mathcal{P}_{T}+\sum_{T \in \mathbb{T}}\left(\sum_{S<T} \phi_{S} \psi_{T \backslash T^{\prime}}(t)\right) \mathcal{P}_{T} \\
& =\sum_{T \in \mathbb{T}}\left(\phi_{T}+\sum_{S<T} \phi_{S} \psi_{T \backslash T^{\prime}}(t)\right) \mathcal{P}_{T} .
\end{aligned}
$$

Comparing this with (3.17), and again employing Corollary 2.6, we get (3.15).

An interesting consequence of the proposition above is the following recurrence formula for $\phi_{T}$ in terms of the number of the leaves of $T$.

Proposition 3.14. For any rooted tree $T$, suppose that the root $r t_{T}$ has $d$ children, i.e., $d=\left|r t_{T}^{+}\right|$. Then

$$
\sum_{r=0}^{l(T)} \sum_{\substack{\left\{v_{1}, v_{2}, \ldots, v_{r}\right\} \subseteq L(T) \\ v_{1}, v_{2}, \ldots, v_{r} \text { distinct }}} \phi_{T \backslash\left\{v_{1}, v_{2}, \ldots, v_{r}\right\}}=\delta_{d, 1} \phi_{T \backslash\left\{r t_{T}\right\}} .
$$

Proof. From (3.14), setting $t=1$, we get

$$
\psi_{T}^{\prime}(1)= \begin{cases}\phi_{T}+\phi_{T \backslash\left\{\mathrm{rt}_{T}\right\}} & \text { if } \quad d=1, \\ \phi_{T} & \text { if } d \geq 2\end{cases}
$$

since, by Lemma $3.9 \psi_{T_{2}}(1)=0$ except when $T_{2}$ is the singleton. From (3.15), setting $t=1$, we get

$$
\psi_{T}^{\prime}(1)=\phi_{T}+\sum_{r=1}^{l(T)} \sum_{\substack{\left\{v_{1}, v_{2}, \ldots, v_{r}\right\} \subseteq L(T) \\ v_{1}, v_{2}, \ldots, v_{r} \text { distinct }}} \phi_{T \backslash\left\{v_{1}, v_{2}, \ldots, v_{r}\right\}}
$$

since $\psi_{T \backslash S}(1)=0$ except when $T \backslash S$ is the disjoint union of finitely many singletons. Comparing (3.19) and (3.20) gives (3.18).

Before leaving this subsection, we will do some calculations on the polynomials $\psi_{T}(t)$ for the chains $C_{n}$ and shrubs $S_{n}$.

Consider the generating functions $\mathrm{C}(t, x)=\sum_{n=0}^{\infty} \psi_{C_{n}}(t) x^{n}\left(\right.$ set $\left.\psi_{C_{0}}(t)=1\right)$ and $\mathcal{S}(t, x)=\sum_{n=0}^{\infty} \psi_{S_{n}}(t) \frac{x^{n}}{n !}$.

Corollary 3.15. The generating functions $\mathrm{C}(t, x)$ and $\mathcal{S}(t, x)$ are given by

$$
\mathcal{C}(t, x)=\exp (t \ln (1+x))=(1+x)^{t}
$$

or, in other words,

$$
\psi_{C_{n}}(t)=\left(\begin{array}{l}
t \\
n
\end{array}\right)=\frac{t(t-1) \cdots(t-n+1)}{n !} .
$$


(b)

$$
\mathcal{S}(t, x)=\frac{e^{x t}-1}{e^{x}-1}
$$

Proof. (a) By Theorem 3.8 and Corollary 3.5 we have

$$
\begin{aligned}
\mathcal{C}(t, x) & =1+\sum_{n=1}^{\infty} \sum_{k=1}^{v\left(C_{n}\right)} \frac{t^{k}}{k !} \sum_{\substack{\bar{e}=\left(e_{1}, \ldots, e_{k-1}\right) \in E\left(C_{n}\right)^{k-1} \\
e_{1} \succ \cdots \succ e_{k-1}}} \phi_{T_{e, 1}} \phi_{T_{e, 2}} \cdots \phi_{T_{e, k}} x^{n} \\
& =1+\sum_{n=1}^{\infty} \sum_{k=1}^{n} \frac{t^{k}}{k !} \sum_{\substack{\left(m_{1}, m_{2}, \ldots, m_{k}\right) \in\left(\mathbb{Z}^{+}\right)^{k} \\
m_{1}+m_{2}+\cdots+m_{k}=n}} \frac{(-1)^{m_{1}}}{m_{1}} \frac{(-1)^{m_{2}}}{m_{2}} \cdots \frac{(-1)^{m_{k}}}{m_{k}} x^{n} \\
& =e^{t\left(-x+\frac{x^{2}}{2}-\cdots+\frac{(-x)^{m}}{m}+\cdots\right)} \\
& =\exp (t \ln (1+x)) .
\end{aligned}
$$

(b) Similarly, we have

$$
\mathcal{S}(t, x)=\sum_{n=0}^{\infty} \sum_{k=1}^{v\left(S_{n}\right)} \frac{t^{k}}{k !} \sum_{\substack{\bar{e}=\left(e_{1}, \ldots, e_{k-1}\right) \in E\left(S_{n}\right)^{k-1} \\ e_{1} \succ \cdots \succ e_{k-1}}} \phi_{T_{e, 1}} \phi_{T_{e, 2}} \cdots \phi_{T_{e, k}} \frac{x^{n}}{n !}
$$

Noting that all but one of $\phi_{T_{e, 2}}, \ldots, \phi_{T_{e, k}}$ are singletons, the remaining one being $S_{n-k+1}$ :

$$
\begin{aligned}
& =\sum_{n=0}^{\infty} \sum_{k=1}^{n+1} \frac{t^{k}}{k !}(k-1) !\left(\begin{array}{c}
m \\
k-1
\end{array}\right) b_{n-k+1} \frac{x^{n}}{n !} \\
& =x^{-1} \sum_{n=0}^{\infty} \sum_{k=1}^{n+1} \frac{(x t)^{k}}{k !} b_{n-k+1} \frac{x^{n-k+1}}{(n-k+1) !} .
\end{aligned}
$$

Replacing $n$ by $n-1$ :

$$
\begin{aligned}
& =x^{-1} \sum_{n=1}^{\infty} \sum_{k=1}^{n} \frac{(x t)^{k}}{k !} b_{n-k} \frac{x^{n-k}}{(n-k) !} \\
& =x^{-1}\left(e^{x t}-1\right) \frac{x}{e^{x}-1} \\
& =\frac{e^{x t}-1}{e^{x}-1} .
\end{aligned}
$$

Remark 3.16. The formulas of Corollary 3.15 can also be easily derived from Theorem 4.2 in the next section. But we think the calculations above are more intriguing.

\section{The Main Theorem}

4.1. Main Theorem on $\psi_{T}(t)$. In the last section, we defined the polynomial $\psi_{T}(t)$, for each rooted tree $T$ (see Theorem 3.8). For each rooted forest $P$, i.e., the disjoint union of finitely many rooted trees $T_{i}(i=1,2, \ldots, k)$, we also defined $\psi_{P}$ 
(see Definition (3.10). Recalling from $\S 2.1$ the definition of a rooted subtree, we are now ready to prove the following main theorem.

Theorem 4.1. Let $t$ and $s$ be indeterminates. For $T \in \mathbb{T}$ we have

$$
\psi_{T}(t+s)=\psi_{T}(t)+\psi_{T}(s)+\sum_{T^{\prime}<T} \psi_{T \backslash T^{\prime}}(t) \psi_{T^{\prime}}(s)
$$

where the last sum runs over all proper rooted subtrees $T^{\prime}$ of $T$.

Proof. Clearly $\exp ((t+s) A) \cdot z=\exp (t A) \cdot \exp (s A) \cdot z$. So we have

$$
\begin{aligned}
z+\sum_{T \in \mathbb{T}} \psi_{T}(t+s) \mathcal{P}_{T} & =\exp ((t+s) A) \cdot z=\exp (t A) \cdot \exp (s A) \cdot z \\
& =\exp (t A) \cdot\left(z+\sum_{T \in \mathbb{T}} \psi_{T}(s) \mathcal{P}_{T}\right) \\
& =\exp (t A) \cdot z+\exp (t A) \cdot\left(\sum_{T \in \mathbb{T}} \psi_{T}(s) \mathcal{P}_{T}\right) \\
& =z+\sum_{T \in \mathbb{T}} \psi_{T}(t) \mathcal{P}_{T}+\sum_{T \in \mathbb{T}} \psi_{T}(s)\left(\exp (t A) \cdot \mathcal{P}_{T}\right)
\end{aligned}
$$

Applying Theorem 3.12 to $\exp (t A) \cdot \mathcal{P}_{T}$ :

$$
\begin{aligned}
& =z+\sum_{T \in \mathbb{T}} \psi_{T}(t) \mathcal{P}_{T}+\sum_{T \in \mathbb{T}} \psi_{T}(s)\left(\mathcal{P}_{T}+\sum_{S \in \mathbb{T}}\left(\sum_{\substack{S^{\prime}<S \\
S^{\prime} \cong T}} \psi_{S \backslash S^{\prime}}(t)\right) \mathcal{P}_{S}\right) \\
& =z+\sum_{T \in \mathbb{T}} \psi_{T}(t) \mathcal{P}_{T}+\sum_{T \in \mathbb{T}} \psi_{T}(s) \mathcal{P}_{T}+\sum_{S \in \mathbb{T}}\left(\sum_{T \in \mathbb{T}} \sum_{\substack{S^{\prime} \leq S \\
S^{\prime} \cong T}} \psi_{S \backslash S^{\prime}}(t) \psi_{T}(s)\right) \mathcal{P}_{S} \\
& =z+\sum_{T \in \mathbb{T}} \psi_{T}(t) \mathcal{P}_{T}+\sum_{T \in \mathbb{T}} \psi_{T}(s) \mathcal{P}_{T}+\sum_{S \in \mathbb{T}} \sum_{S^{\prime}<S} \psi_{S \backslash S^{\prime}}(t) \psi_{S^{\prime}}(s) \mathcal{P}_{S} .
\end{aligned}
$$

Replacing $S$ by $T$ in the last summation:

$$
=z+\sum_{T \in \mathbb{T}} \psi_{T}(t) \mathcal{P}_{T}+\sum_{T \in \mathbb{T}} \psi_{T}(s) \mathcal{P}_{T}+\sum_{T \in \mathbb{T}} \sum_{T^{\prime}<T} \psi_{T \backslash T^{\prime}}(t) \psi_{T^{\prime}}(s) \mathcal{P}_{T} .
$$

According to Corollary 2.6 and an easy specialization argument, the theorem follows by comparing the coefficients of $\mathcal{P}_{T}$ in the above equation.

The difference polynomial of $g(t) \in \mathbb{C}[T]$ is defined to be the polynomial $\Delta g(t)=$ $g(t+1)-g(t)$. The following special case of the theorem above, which gives a formula for the difference polynomial of $\psi_{T}(t)$, is most useful to us.

Theorem 4.2. For any tree $T$ with $v(T) \geq 2$, we have

$$
\begin{aligned}
\Delta \psi_{T}(t) & =\psi_{T_{1}}(t) \psi_{T_{2}}(t) \cdots \psi_{T_{d}}(t) \\
& =\psi_{T \backslash\left\{r t_{T}\right\}}(t)
\end{aligned}
$$

where $T_{i}, i=1,2, \ldots, d$ are the connected components of $T \backslash\left\{r t_{T}\right\}$. 
Proof. This follows form Theorem 4.1 by setting $s=1$ in (4.1) and appealing to Lemma 3.9, which says that $\psi_{T}(1)=0$ unless $T$ is the singleton, in which case $\psi_{T}(1)=1$.

Theorem 4.3. For any tree $T$ with $v(T) \geq 2$, we have

$$
\Delta \psi_{T}(t)=\sum_{r=1}^{l(T)} \sum_{\substack{\left\{v_{1}, v_{2}, \ldots, v_{r}\right\} \subseteq L(T) \\ v_{1}, v_{2}, \ldots, v_{r} \text { distinct }}} \psi_{T \backslash\left\{v_{1}, v_{2}, \ldots, v_{r}\right\}}(t) .
$$

$$
\psi_{T \backslash\left\{r t_{T}\right\}}(t)=\sum_{r=1}^{l(T)} \sum_{\substack{\left\{v_{1}, v_{2}, \ldots, v_{r}\right\} \subseteq L(T) \\ v_{1}, v_{2}, \ldots, v_{r} \text { distinct }}} \psi_{T \backslash\left\{v_{1}, v_{2}, \ldots, v_{r}\right\}}(t)
$$

where $T_{i}, i=1,2, \ldots, d$ are the connected components of $T \backslash\left\{r t_{T}\right\}$.

Proof. Clearly, (b) follows from (a) and Theorem 4.2 For (a), switch $t$ and $s$ and set $s=1$ in 4.1 to get

$$
\psi_{T}(t+1)=\psi_{T}(t)+\psi_{T}(1)+\sum_{T^{\prime}<T} \psi_{T \backslash T^{\prime}}(1) \psi_{T^{\prime}}(t) .
$$

By Lemma 3.9, we have $\psi_{T}(1)=0$ and $\psi_{T \backslash T^{\prime}}(1)=0$, unless $T \backslash T^{\prime}$ is a disjoint union of singletons, in which case $\psi_{T \backslash T^{\prime}}(1)=1$. Therefore,

$$
\psi_{T}(t+1)-\psi_{T}(t)=\sum_{r=1}^{l(T)} \sum_{\substack{\left\{v_{1}, v_{2}, \ldots, v_{r}\right\} \subseteq L(T) \\ v_{1}, v_{2}, \ldots, v_{r} \text { distinct }}} \psi_{T \backslash\left\{v_{1}, v_{2}, \ldots, v_{r}\right\}}(t)
$$

as desired.

4.2. Algorithm for $\psi_{T}(t)$. From Theorem 4.2 we get the following algorithm for computing $\psi_{T}(t)$. Here, for $h(t) \in \mathbb{C}[t], \Delta^{-1} h(t)$ is defined to be the unique polynomial $g(t) \in \mathbb{C}[t]$ such that $\Delta g(t)=h(t)$ and $g(0)=0$.

Algorithm. For any fixed rooted tree $T$, we sign a polynomial $N_{v}(t)$ to each vertex $v$ of $T$ as follows:

(1) For each leaf $v$ of $T$, set $N_{v}(t)=t$.

(2) For any other vertex $v$ of $T$, define $N_{v}(t)$ inductively starting from the highest level by $N_{v}(t)=\Delta^{-1}\left(N_{v_{1}}(t) N_{v_{2}}(t) \cdots N_{v_{k}}(t)\right)$, where $v_{j}, j=1,2, \ldots, k$, are the distinct children of $v$.

Then for each vertex $v$ of $T, N_{v}(t)=\psi_{T_{v}^{+}}(t)$, where $T_{v}^{+}$is the subtree of $T$ rooted at the vertex $v$. In particular, we have $\psi_{T}(t)=N_{\mathrm{rt}_{T}}(t)$.

The following example applies this algorithm to the shrubs $S_{n}$ to show that the polynomials $\psi_{S_{n}}(t)$ are closely related to the Bernoulli polynomials $B_{n}(t)$ defined by $\frac{x e^{t x}}{e^{x}-1}=\sum_{n=0}^{\infty} B_{n}(t) \frac{x^{n}}{n !}$. (Compare this with (b) of Corollary 3.15.)

Example 4.4. Let $v_{1}, \ldots, v_{n}$ be the leaves of the shrub $S_{n}$. Following the algorithm, we first assign the polynomial $t$ to each leaf $v_{i}$. The next step in the algorithm gives

$$
\psi_{S_{n}}(t)=\Delta^{-1}\left(t^{n}\right)
$$


One of the fundamental properties of the Bernoulli polynomials $B_{n}(t)$ is

$$
\Delta B_{n}(t)=B_{n}(t+1)-B_{n}(t)=n t^{n-1},
$$

and from this and the fact that $\Delta$ commutes with $\frac{d}{d t}$ one easily derives

$$
\frac{d}{d t} B_{n+1}(t)=(n+1) B_{n}(t) .
$$

From (4.5) and (4.6) we get

$$
\Delta^{-1}\left(t^{n}\right)=\int_{0}^{t} B_{n}(u) d u=\frac{B_{n+1}(t)-B_{n+1}(0)}{n+1} .
$$

Putting together equations (4.4) and (4.7), we obtain this relationship between $\psi_{S_{n}}(t)$ and $B_{n+1}(t)$

$$
\psi_{S_{n}}(t)=\int_{0}^{t} B_{n}(u) d u=\frac{B_{n+1}(t)-B_{n+1}(0)}{n+1} .
$$

4.3. Combinatorial Property of $\psi_{T}(t)$. After the main part of this work was done, Professor John Shareshian pointed out to us that the polynomial $\psi_{T}(t)$ for rooted trees coincides with the strict order polynomial $\bar{\Omega}(P, t)$ for finite posets (partial ordered sets) $P$ in combinatorics (see Chapters 3 and 4 in [St1]). We first recall the polynomial $\bar{\Omega}(P, t)$ associated with a finite poset, and then we show that, when $P$ is the poset of the set $V(T)$ of vertices of a rooted tree $T$ with the natural partial order induced by ancestry (the root being the unique smallest element), we have $\psi_{T}(t)=\bar{\Omega}(P, t)$.

Any rooted tree corresponds in this way to a unique finite poset, and a finite poset $P$ corresponds to a rooted tree precisely when it satisfies these two criteria:

(1) $P$ has a unique smallest element, and

(2) any interval in $P$ is totally ordered.

For any $n \in \mathbb{N}$, the chain $C_{n}$ gives the totally ordered poset with $n$ elements. (We can view it as the set $\{1,2, \ldots, n\}$ with the natural order of the positive integers.) For any poset $P$, we say that a map $f: P \rightarrow C_{n}$ is strict order-preserving if $f(a)<f(b)$ in $C_{n}$ whenever $a<b$ in $P$. It is well known that there exists a unique polynomial $\bar{\Omega}(P, t)$ such that $\bar{\Omega}(P, n)$ equals the number of strict order-preserving maps $f$ from $P$ to $C_{n}$ for all $n \in \mathbb{N}$. This, then, is the theorem shown to us by John Shareshian.

Theorem 4.5. For any rooted tree $T$, we have

$$
\psi_{T}(t)=\bar{\Omega}(T, t)
$$

(where, on the right, $T$ is viewed as a finite poset as described above).

Proof. It is obvious that when $T$ is the singleton, $\bar{\Omega}(T, t)=t$. Hence it is enough to show that $\bar{\Omega}(T, t)$ also satisfies the recursion formula of Theorem 4.2 More precisely, we will show that, in the notation of Theorem 4.2 we have

$$
\Delta \bar{\Omega}(T, n)=\bar{\Omega}(T, n+1)-\bar{\Omega}(T, n)=\bar{\Omega}\left(T_{1}, n\right) \bar{\Omega}\left(T_{2}, n\right) \cdots \bar{\Omega}\left(T_{d}, n\right)
$$

for any $n \in \mathbb{N}$.

Note that $\Delta \bar{\Omega}(T, n)$ equals the number of strict order-preserving maps $f$ from $T$ to $C_{n+1}=\{1,2, \ldots, n+1\}$ such that $f\left(\mathrm{rt}_{T}\right)=1$. But this number is also the same as the number of strict order-preserving maps $g$ from $T \backslash\left\{\mathrm{rt}_{T}\right\}$ to $C_{n}$, which is $\bar{\Omega}\left(T_{1}, n\right) \bar{\Omega}\left(T_{2}, n\right) \cdots \bar{\Omega}\left(T_{d}, n\right)$. 
Remark 4.6. It is interesting that the strict order polynomial $\bar{\Omega}(T, t)$ for the finite posets induced by rooted trees $T$ can be defined in a totally different way, namely, according to the formula (3.11) of Theorem 3.8. In fact, this realization of the strict order polynomial can be generalized to an arbitrary finite poset $P$. This generalization and its consequences will be discussed in the upcoming paper [SWZ].

\section{Some Applications}

For a formal automorphism $F=\left(F_{1}, F_{2}, \ldots, F_{n}\right)=z+H$ of the form identity plus higher, we give a restatement and new proof of the tree formula for the formal inverse first proved in $[\mathrm{BCW}]$ and $[\mathrm{W} 2$.

Theorem 5.1. For any rooted tree $T$, we have $\psi_{T}(-1)=(-1)^{v(T)}$. Hence the formal inverse $F^{-1}$ of $F$ is given by

$$
F^{-1}=z+\sum_{T \in \mathbb{T}}(-1)^{v(T)} \mathcal{P}_{T} .
$$

Proof. The formula (5.1) follows from $\psi_{T}(-1)=(-1)^{v(T)}$ by Proposition 3.7 and Theorem 3.8 .

It is well known in combinatorics (see [St1]) that the strict order polynomials satisfy $\bar{\Omega}(T,-1)=(-1)^{v(T)}$, from which the result follows, in light of Theorem 4.5 For completeness, we give a direct proof here.

We use the mathematical induction on $v(T)$. The case for $v(T)=1$ is trivial. $\left(\psi_{T}(t)=t\right.$ in this case.) Suppose $v(T) \geq 2$. By Theorem 4.2 setting $t=-1$, we have

$$
\psi_{T}(0)-\psi_{T}(-1)=\psi_{T_{1}}(-1) \psi_{T_{2}}(-1) \cdots \psi_{T_{d}}(-1)
$$

where $T_{i}, i=1,2, \ldots, d$ are the connected components of $T \backslash\left\{\operatorname{rt}_{T}\right\}$. We have $\psi_{T}(0)=0$, and by induction we may assume the theorem holds for $T_{1}, \ldots, T_{d}$. Hence

$$
\psi_{T}(-1)=-(-1)^{v_{T_{1}}+v_{T_{2}}+\cdots+v_{T_{d}}}=(-1)^{v(T)} .
$$

It is known that the Jacobian conjecture (see BCW] for a statement of this famous problem) is equivalent to the assertion that

$$
\sum_{T \in \mathbb{T}_{N}} \mathcal{P}_{T}=0
$$

for $N>>0$ whenever $H$ is a homogeneous polynomial system (of degree $\geq 2$ ) and the Jacobian determinant $\left|\left(D_{j} F_{i}\right)\right|$ is (everywhere) nonzero. In fact, this follows from Theorem 5.1, since when $H$ is homogeneous the polynomials $\sum_{T \in \mathbb{T}_{N}} \mathcal{P}_{T}$, for fixed $N$, are the homogeneous summands of $F^{-1}$ (see Remark 2.2). When $H$ is homogeneous, the condition $\left|\left(D_{j} F_{i}\right)\right|=1$ is known to be equivalent to the nilpotence of the Jacobian matrix $J H=\left(D_{j} H_{i}\right)$ (see [BCW]). Thus the following result presents an intriguing statement for comparison.

\footnotetext{
${ }^{4} \mathrm{The}$ formula as given in $[\mathrm{BCW}]$ and $[\mathrm{W} 2]$ did not include the factor $(-1)^{v(T)}$. It appears here because of our choice in writing $F=z+H$ instead of $F=z-H$.
} 
Proposition 5.2. Assume $H$ is homogeneous of degree $\geq 2$. For any rooted tree, let $h_{T, k}$ be the number of vertices of height $k$. Suppose that $(J H)^{k}=0$. Then

$$
\sum_{T \in \mathbb{T}_{N}} h_{T, m} \mathcal{P}_{T}=0
$$

for any $N \in \mathbb{N}$ and $m \geq k$.

Proof. Suppose that $\operatorname{deg} H=d \geq 2$. It follows from Euler's formula that $J H \cdot\left(z^{\mathrm{t}}\right)=$ $(d H)^{\mathrm{t}}$, from which we get $\frac{1}{d}(J H)^{k} \cdot\left(z^{\mathrm{t}}\right)=(J H)^{k-1} \cdot H^{\mathrm{t}}=0$. (Here the superscript ${ }^{\mathrm{t}}$ denotes transpose, converting a row to a column so that the matrix multiplications make sense.) For any integer $m \geq 1$, a straightforward calculation shows that the chain $C_{m}$ has the property $\mathcal{P}_{C_{m}}=J H^{m-1} \cdot H^{\mathrm{t}}$. Therefore, $\mathcal{P}_{C_{m}}=0$ for $m \geq k$, and we have

$$
0=\exp (-A) \cdot \mathcal{P}_{C_{m}} .
$$

By Theorem 3.12, setting $S=C_{m}$ and $t=-1$ in (3.13):

$$
=\sum_{T \in \mathbb{T}}\left(\sum_{\substack{T^{\prime} \leq T \\ T^{\prime} \cong C_{m}}} \psi_{T \backslash T^{\prime}}(-1)\right) \mathcal{P}_{T} .
$$

By Theorem 5.1:

$$
\begin{aligned}
& =\sum_{T \in \mathbb{T}}\left(\sum_{\substack{T^{\prime} \leq T \\
T^{\prime} \cong C_{m}}}(-1)^{v\left(T \backslash T^{\prime}\right)}\right) \mathcal{P}_{T} \\
& =\sum_{T \in \mathbb{T}}(-1)^{v(T)-m} h_{T, m} \mathcal{P}_{T} .
\end{aligned}
$$

In particular, for any $N \in \mathbb{N}$, we have

$$
\sum_{T \in \mathbb{T}_{N}}(-1)^{N-m} h_{T, m} \mathcal{P}_{T}=(-1)^{N-m} \sum_{T \in \mathbb{T}_{N}} h_{T, m} \mathcal{P}_{T}=0,
$$

which gives (5.3).

The proposition above shows that, for a fixed homogeneous polynomial system $H$, the polynomials $\mathcal{P}_{T}$ are in some sense quite linearly dependent on each other.

Finally, let us point out that the formal flow $F_{t}$ gives a formal flow between $F$ and the identity map id, i.e., $\left.F_{t}\right|_{t=1}=F$ and $\left.F_{t}\right|_{t=0}=$ id, having the additional properties $F_{t}(0)=0$ and $J F_{t}(0)=I_{n}$. It is an open question in complex analysis whether, for any local analytic map $F$, such an analytic flow exists. The usual approach to this question is to show that $F$ is linearizable, i.e., it is conjugate to a linear map. But when $F$ is linearizable the question is still open, even for the one-variable case. (There are many partial results for this problem.) So it is of interest that the formal solution to this question is given by the very clean formula (3.10) of Theorem [3.8. But the question of when $F_{t}$ is locally convergent is still open. 


\section{REFERENCES}

[A] S. S. Abhyankar, Lectures in algebraic geometry, Notes by Chris Christensen, Purdue Univ., 1974.

[BCW] H. Bass, E. Connell, and D. Wright, The Jacobian conjecture, reduction of degree and formal expansion of the inverse. Bull. Amer. Math. Soc. 7, (1982), 287-330. MR 83k:14028. Zbl.539.13012.

[CMTWW] C. C.-A. Cheng, J. H. McKay, J. Towber, S. S.-S. Wang, and D. Wright, Reversion of power series and the extended Raney coefficients, Trans. Amer. Math. Soc. 349 (1997), 1769-1782. MR 97h:13018 Zbl.868.13019.

[Ge] I. M. Gessel, A combinatorial proof of the multivariable Lagrange inversion formula, J. Combin. Theory Ser. A, 45 (1987), 178-195. MR 88h:05011 Zbl.651.05009.

[Go] I. J. Good, Generalizations to several variables of Lagrange's expansion, with applications to stochastic processes, Proc. Cambridge Philos. Soc. 56 (1960), 367-380. MR 23:A352 Zbl.135.18802.

[HS] M. Haiman and W. Schmitt, Incidence algebra antipodes and Lagrange inversion in one and several variables, J. Combin. Theory Ser. A, 50 (1989), 172-185. MR 90f:05005 Zbl.747.05007.

[Ja] C. G. J. Jacobi, De resolutione aequationum per series infinitas, J. Reine Angew. Math. 184 (1830), 257-286.

[Jo] S. A. Joni, Lagrange inversion in higher dimensions and umbral operators, Linear and Multilinear Algebra 6 (1978) 111-122. MR 58:10485 Zbl.395.05005.

[R] G. N. Raney, Functional composition patterns and power series reversion, Trans. Amer. Math. Soc. 94 (1960), 441-451. MR 22:5584 Zbl.131,14.

[St1] Richard P. Stanley, Enumerative Combinatorics I, Cambridge University Press, 1997. MR 98a:05001 Zbl.945.05006.

[St2] Richard P. Stanley, Enumerative Combinatorics II, Cambridge University Press, 1999. MR 2000k:05026 Zbl.928.05001.

[Sh] J. Shareshian, Personal communication.

[SWZ] J. Shareshian, D. Wright and W. Zhao, A New Realization of Order Polynomials. In Preparation.

[W1] D. Wright, Formal inverse expansion and the Jacobian Conjecture, J. Pure Appl. Algebra, 48 (1987), 199-219. MR 89b:13008 Zbl.666.12017.

[W2] D. Wright, The tree formulas for reversion of power series, J. Pure Appl. Algebra, 57 (1989) 191-211. MR 90d:13008 Zbl.672.13010.

[Z] W. Zhao, Exponential formulas for the Jacobians and Jacobian matrices of analytic maps, J. Pure Appl. Algebra, 166 (2002) 321-336. MR 2002i:14059

Department of Mathematics, Washington University in St. Louis, St. Louis, Missouri 63130-4899

E-mail address: wright@einstein.wustl.edu

Department of Mathematics, Washington University in St. Louis, St. Louis, Missouri 63130-4899

E-mail address: zhao@math. wustl.edu 